
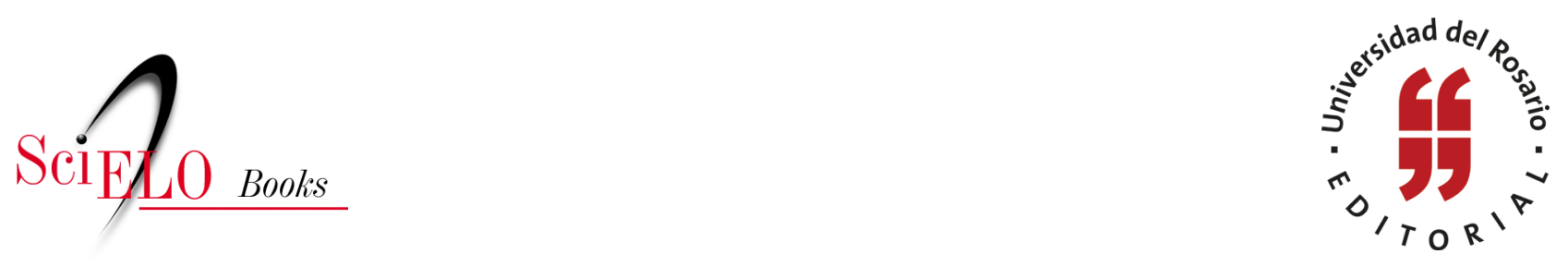

\title{
Capítulo 5. Modelos de estratificación socioeconómica a partir de la información catastral para la ciudad de Bogotá, D.C.
}

\author{
Denis López \\ Carlos E. Sepúlveda
}

\section{SciELO Books / SciELO Livros / SciELO Libros}

LÓPEZ, D., and SEPÚLVEDA, C.E. Modelos de estratificación socioeconómica a partir de la información catastral para la ciudad de Bogotá, D.C. In: SEPÚLVEDA RICO, C.E., LÓPEZ CAMACHO, D., and GALLEGO ACEVEDO, J.M., eds. Los límites de la estratificación: en busca de alternativas [online]. Bogotá: Editorial Universidad del Rosario: Alcaldía Mayor de Bogotá D.C., 2014, pp. 109-147. ISBN: 978-958-738-537-3. https://doi.org/10.7476/9789587385373.0007.

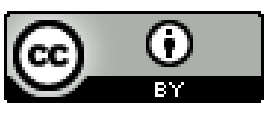

All the contents of this work, except where otherwise noted, is licensed under a Creative Commons Attribution 4.0 $\underline{\text { International license. }}$

Todo o conteúdo deste trabalho, exceto quando houver ressalva, é publicado sob a licença Creative Commons Atribição 4.0.

Todo el contenido de esta obra, excepto donde se indique lo contrario, está bajo licencia de la licencia Creative Commons Reconocimento 4.0 . 


\section{Capítulo 5 \\ Modelos de estratificación socioeconómica a partir de la información catastral para la ciudad de Bogotá, D.C.}

Denis López y Carlos E. Sepúlveda**

Palabras clave: modelos de estratificación, clasificación predial, focalización del gasto público, subsidios en servicios públicos domiciliarios.

\section{Introducción}

Soportado en datos cartográficos y de atributos asociados (jurídicos, físicos, de dotación de recursos y de valor comercial), que se gestionan mediante sistemas de información geográfica, el sistema de información catastral se compone de un conjunto de 'variables' relativas al registro y la propiedad del suelo, el inventario detallado de las características del suelo y las construcciones (aspecto físico), y la zonificación de áreas de homogeneidad física y económica. La información que maneja dicho sistema sintetiza y cristaliza la diferenciación socioeconómica existente en lotes, construcciones y características arquitectónicas, localizaciones geográficas, condiciones urbanísticas, usos del suelo y entornos específicos en el interior de la ciudad. Tal diferen-

* Consultor asociado Infométrika, SAS.

** Profesor principal, Facultad de Economía de la Universidad del Rosario. 
ciación de la localización, tipo de construcción y condiciones de entorno de la vivienda conforman indicadores altamente correlacionados con diversas aproximaciones a la diferenciación económica y social de los residentes de la ciudad (Bonilla et al., cap. 2; Bernal et al., cap. 3; y Acosta et al., cap. 4).

La diferenciación de los predios, y, entre ellos, los de uso residencial conectados a las redes de servicios públicos domiciliarios, puede realizarse a partir de la información catastral relativa a las características físicas (puntajes de construcción detallada o totalizada) y características de precios de mercado tanto del suelo como de las construcciones en él localizadas. A lo largo de este libro, se ha propuesto el valor unitario integral del avalúo (VUI) como la variable que recoge los precios (y características) tanto del suelo como de la construcción del inmueble (cap. 3). Adicionalmente, se ha presentado evidencia de que es este valor unitario integral el que se correlaciona de una manera más estrecha con las características socioeconómicas medidas a través de distintos indicadores de capacidad de pago y bienestar de los hogares (cap. 4).

El objetivo principal de este capítulo es estudiar el impacto de la información catastral al ser tenido en cuenta como elemento base de la estratificación socioeconómica de la ciudad de Bogotá, D.C., para el manejo de subsidios y contribuciones dentro de la política redistributiva en SPD, y desarrollar un conjunto de ejercicios que analicen la idoneidad y eficacia de estratificar o clasificar los inmuebles residenciales con las variables que provee el sistema catastral de la ciudad.

De esta forma, el capítulo presenta modelos alternativos de estratificación de bienes inmuebles en el Distrito Capital, utilizando como fuente primaria el catastro, y explora eventuales impactos en su implementación. El ejercicio se realiza desde dos perspectivas: una nacional, partiendo de resultados previos del DANE (2011), que relaciona información de la muestra cocensal 2005 con información catastral a nivel nacional; y una segunda toma al Distrito Capital aparte, utilizando directamente el avalúo catastral (valor unitario integral) como variable básica de clasificación. Se explora adicionalmente la posibilidad de obtener un número de estratos óptimo de manera endógena para Bogotá, que resulta en esta oportunidad en nueve grupos.

Los impactos de estas nuevas metodologías se estiman desde el punto de vista de cambio en la distribución de estratos, movimiento de estratos de los hogares, errores de inclusión, estimando el balance del sistema cruzado de subsidios (impacto financiero). 
Como resultado, se encuentra que, a pesar de que una eventual estratificación socioeconómica basada en información catastral presenta una mejora notable en los errores de inclusión, existen limitaciones respecto a variaciones muy fuertes en los estratos asignados (que dificultaría su implementación) o a la presencia aún de errores de inclusión significativos. Esto lleva a plantear la posibilidad de explorar esquemas alternativos, diferentes a la estratificación socioeconómica, para el sistema subsidiario de pago de SPD. Particularmente, considerar directamente el avalúo catastral para establecer la tarifa de pago de SPD sin necesidad de establecer estratos.

\subsection{Contexto general}

De los capítulos anteriores, se recogen los siguientes elementos:

1) La estratificación socioeconómica es un instrumento de clasificación de inmuebles residenciales urbanos con miras a la gestión de un sistema cruzado de subsidios y contribuciones en la prestación de los SPD.

2) Aunque la estratificación es una clasificación de inmuebles residenciales y no de hogares, debe buscarse aproximaciones cada vez mejores para correlacionar o encontrar correspondencias entre variables de tipo socioeconómico, como los ingresos, gastos, capacidad de pago y calidad de vida, de un lado; y características de las viviendas y del entorno, del otro, y, de esta manera, fundamentar la estratificación de inmuebles residenciales para la focalización de subsidios y el cobro de contribuciones a los usuarios de SPD (DANE, 2011).

3) Con el paso de los años, al mantenerse estática, la metodología de estratificación desencadena problemas evidentes. Errores de inclusión significativos (inmuebles residenciales clasificados en estratos 1, 2 y 3 incluyen hogares que tienen altos ingresos y reciben subsidios para SPD) indican que la estratificación no refleja de manera óptima la diferenciación de grupos a partir de su capacidad económica; riesgos en la sostenibilidad del sistema solidario de pagos de SPD, en gran parte por estos errores de inclusión; posibilidades de debilidades técnicas de recolección de la información que alimenta el modelo de estratificación, ya que se realiza de forma separada por parte de cada municipio a partir de observación de variables por predominancia, entre otros. 
4) El catastro inmobiliario constituye un sistema integrado que reúne información técnica y objetiva para la totalidad de los predios de la ciudad. El valor unitario integral, definido como la sumatoria de valores unitarios de suelo y construcción, para los predios no sujetos al régimen de propiedad horizontal, y avalúo catastral sobre el área construida, para aquellos que están sujetos al régimen de propiedad horizontal, constituye una base idónea para la estratificación de los inmuebles residenciales.

5) Las variables catastrales, y en particular el VUI, expresan una importante proporción de la varianza explicada por los indicadores socioeconómicos. La correlación de las variables catastrales con las indicadores socioeconómicos resulta consistente con la conceptualización desarrollada en el capítulo 3, que sustenta que, por más variables detalladas que se puedan utilizar, los precios del suelo -referente de las rentas diferenciales que se producen y se pagan en la ciudad- son una síntesis que representa las muy diversas condiciones de diferenciación urbana, desde la tipología constructiva, pasando por la diferenciación arquitectónica, urbanística, geográfica y de entorno; y hasta la existencia y calidad de los bienes públicos, de la infraestructura de servicios, la vialidad y conectividad, la calidad de vida y las condiciones diferenciales de bienestar humano y social, cualquiera que pueda ser su forma de medición.

6) En la estimación oficial del valor unitario de la construcción, se incorpora explícitamente el estrato de la vivienda, lo que lleva a una influencia de la estratificación actual en la conformación del avalúo catastral. En este sentido, una futura estratificación con base en información catastral, y en especial el avalúo, se vería afectada por la estratificación anterior (la vigente en la actualidad), lo que hace necesario eliminar ese efecto para estimar el impacto o su pertinencia como elemento clasificador de inmuebles.

\subsection{Modelos de estratificación socioeconómica con base en variables catastrales}

De acuerdo con los argumentos expuestos, esta sección incorpora la información catastral en diferentes modelos de estratificación socioeconómica para 
el Distrito Capital. Se pretende así construir un procedimiento que clasifique inmuebles a partir de la mejor información posible.

El propósito general es desarrollar modelos que conformen un número definido de grupos (seis en el marco de la Ley 142 de 1994), que sean conjuntos homogéneos de domicilios, lo más claramente diferenciados entre sí, de manera que facilite la aplicación de subsidios y contribuciones en las tarifas de SPD, disminuyendo los errores de inclusión actuales.

Este ejercicio se efectúa desde dos grandes perspectivas. Una primera parte de una concepción de un sistema solidario de pagos de SPD a nivel nacional, que lleva a considerar definiciones y metodologías de estratificación a esa escala. Esto tiene una lógica desde el punto de vista de diseño de política de subsidios tarifarios, considerando que para algunos SPD el sistema cruzado se establece dentro de una concepción nacional (p. ej., electricidad).

En este sentido, a partir del trabajo desarrollado por el DANE (2011), se toman modelos que relacionan información de características socioeconómicas (ingreso, calidad de vida urbana, gasto corriente) de la muestra cocensal 2005 y encuestas de calidad de vida, con la información de predios que contienen las fichas de los catastros nacionales. Estos ejercicios resultan en modelos de estratificación para diferentes tipologías de ciudades, que ponderan variables contenidas en la ficha catastral (fuertemente correlacionadas en una etapa previa con las características de los hogares) y resultan en una asignación de estrato a cada predio domiciliario. Debido a la complejidad urbana relativa de la ciudad de Bogotá, D.C., se definió como una categoría particular de la tipología.

Una segunda perspectiva estudia al Distrito Capital de manera aislada con respecto al resto del país. Es decir, la definición de estratos surge tomando como universo únicamente la población distrital (y no la nacional). Esta perspectiva tiene sentido al considerar que el desarrollo técnico y tecnológico del catastro distrital y de su información es muy superior respecto a los demás catastros de Colombia, lo que eventualmente facilitará, respecto al resto del país, una transición del esquema de estratificación actual a otro que tome la ficha catastral como insumo primario para definir esquemas de subsidios y contribuciones de SPD. Adicionalmente, la discusión de política pública social lleva a plantearse la posibilidad de explorar alternativas diferenciadas de política que respondan a la evolución, diferenciación y complejidad de las dinámicas urbanas respectivas. 


\subsubsection{MODELO DE ESTRATIFICACIÓN BASE NACIONAL, APOYADO EN INFORMACIÓN CATASTRAL}

El DANE (2011) buscó obtener una estratificación estableciendo modelos que asignen a los inmuebles su respectivo estrato, a partir de las variables contenidas en la ficha catastral, siendo estas a su vez un reflejo de las condiciones socioeconómicas de los hogares. Fundamentalmente, busca establecer lo siguiente:

$$
E_{i}=f(\text { variables catastrales } \mid \text { condiciones socioeconómicas })
$$

donde $f($.$) es una función de enlace tipo probit que permite establecer la$ probabilidad de pertenecer a un estrato.

A nivel nacional, aparte de no contar con una base completa y actualizada, existen diferencias importantes en el porcentaje de avalúo comercial adoptado por los distintos municipios en el país, que no hacen posible dentro de esta investigación la construcción del vUi para todo el territorio. Esto nos lleva a tomar directamente los modelos de clasificación explorados por el DANE, los cuales toman la información de la ficha catastral y, a partir de diferentes ponderaciones de sus variables (dependiendo de la tipología de la ciudad), establece estratos para cada bien inmueble.

El diseño metodológico del DANE (2011) tiene dos momentos que se ilustran en la figura 5.1. Una primera etapa establece una clasificación de hogares a través del método Dalenius-Hodges, que, tomando las condiciones socioeconómicas, medidas por tres indicadores diferentes (índice de bienestar socioeconómico ${ }^{1}$, distancia al extremo ${ }^{2}$, capacidad de pago $\left.{ }^{3}\right)$, conforman los 'estratos robustos'.

Los indicadores socioeconómicos se construyen con la información de la muestra cocensal del Censo nacional 2005 y con la Encuesta de calidad de vida 2008. El método de clasificación sobre los indicadores se genera a nivel

1 El IBS es un índice multidimensional que incorpora indicadores referidos a funcionamientos. Para más detalle, ver anexo 3 .

2 Este indicador es una medida de intervalo construida como la distancia a un individuo hipotético que tuviese las peores condiciones observables en la muestra ampliada del Censo 2005.

3 Se utiliza el logaritmo de la capacidad de pago, construida a partir del gasto total menos el consumo en alimentos (Econometría, 2006). 
de manzana para la muestra correspondiente a Bogotá, debido a que, por su complejidad urbanística, la ciudad presenta la posibilidad de tener viviendas de cada uno de los seis estratos socioeconómicos, que terminen de referencia para el resto del país.

Con el objeto de obtener una muestra de aprendizaje, el método de clasificación Dalenius-Hodges ${ }^{4}$ permite generar cinco puntos de corte para cada uno de los tres índices, clasificando así en seis grupos, los cuales se aplican a las demás tipologías de ciudad (se definen seis tipologías de ciudades dependiendo de su complejidad urbanística y socioeconómica), conformando asî una muestra total estratificada a nivel de manzana. Un 'estrato robusto x' se define finalmente por aquellos hogares que hacen parte del grupo ' $x$ ' para cada una de las clasificaciones de los tres índices. Es decir, los hogares que conforman el estrato robusto 1 son aquellos que, sin importar el índice utilizado como base para la clasificación, se encuentran en el primer grupo de los seis.

En un segundo momento de la metodología, se asocia la respectiva información física de la vivienda y del entorno, contenida en la ficha catastral de cada una de las áreas geográficas incluidas en los hogares clasificados en el estrato robusto. Esto permite asociar a las manzanas clasificadas en un estrato determinado en el primer momento, un conjunto de variables de la construcción y de entorno de los predios que pertenecen a dicha manzana.

Con la información observable y disponible en los catastros, se procede a estimar modelos de clasificación (modelos probit acumulativo con respuesta multinomial nominal y ordinal) que asignan el estrato (estrato robusto) en la respectiva tipología de ciudad. Se tienen entonces seis modelos discriminantes (uno para cada tipología de ciudad) que permiten la agrupación (estratificación) a partir de variables explicativas de construcción y entorno tomadas del sistema catastral del país.

$4 \quad$ El método de Dalenius-Hodges busca estratificar una población cuyo coeficiente de variación por estrato sea lo más pequeño posible. 


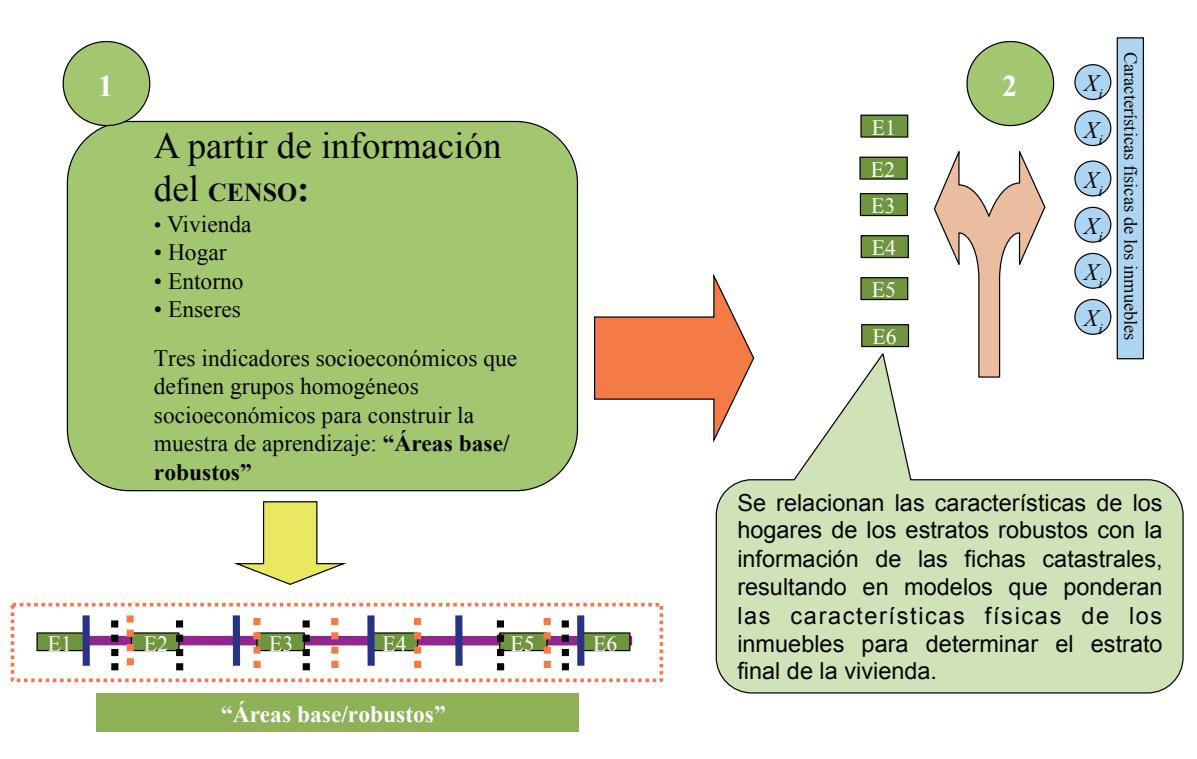

Figura 5.1. Pasos metodológicos DANE (2011)

Fuente: DANE (2011). Propuesta metodológica para la nueva estratificación socioeconómica.

Para nuestro caso, el primer modelo de estratificación es una réplica del método DANE (2011) que resulta para Bogotá, aplicando los parámetros estimados a los predios de la ciudad con base en la información catastral de Bogotá 20125. El modelo final utilizado es:

$$
\varepsilon_{i}=f\left(X_{i}\right)
$$

Donde $X$ son las variables de la ficha catastral que determinan el estrato $\left(\varepsilon_{i}\right)$. Para el caso de Bogotá, las variables discriminantes que resultan y sus respectivos coeficientes se presentan en la tabla 5.1.A. Se tiene entonces que el estrato para cada bien inmueble en la ciudad de Bogotá se determina teniendo en cuenta el puntaje dentro de la ficha catastral de los acabados principales, materiales de cubierta, mobiliario del baño, enchapes de la cocina, valor del metro cuadrado del terreno y de la construcción, y el puntaje residual. Este modelo se referenciará en los resultados como 'modelo base nacional'.

5 Un primer ejercicio buscó implementar la metodología DANE (2011) construyendo el primer momento a partir de la Encuesta multipropósito de Bogotá. Sin embargo, el tamaño de la muestra robusta resultante no permitió tener resultados razonables, en términos de distribuciones de estratos coherentes. 
Tabla 5.1.A. Parámetros estimados para Bogotá

\begin{tabular}{l|c|c|c|c}
\hline \multicolumn{1}{c|}{ Variable } & Parámetro & Parámetro estandarizado & Error estándar & P valor \\
\hline Intercepto 1 & $-1,2$ & & 0,1856 & $<0,0001$ \\
\hline Intercepto 2 & 3,4 & & 0,1771 & $<0,0001$ \\
\hline Intercepto 3 & 7,4 & & 0,1867 & $<0,0001$ \\
\hline Intercepto 4 & 12,5 & & 0,2075 & $<0,0001$ \\
\hline Intercepto 5 & 24,7 & & 0,2562 & $<0,0001$ \\
\hline Acabados principales - pisos & $-0,3$ & $-0,9$ & 0,00841 & $<0,0001$ \\
\hline Estructura - cubierta & $-0,4$ & $-1,6$ & 0,00621 & $<0,0001$ \\
\hline Baño - mobiliario & $-0,4$ & $-1,4$ & 0,0108 & $<0,0001$ \\
\hline Cocina - enchapes & $-0,3$ & $-0,5$ & 0,00897 & $<0,0001$ \\
\hline Valor m ${ }^{2}$ de terreno estandarizado & $-10,6$ & $-16,7$ & 0,1236 & $<0,0001$ \\
\hline Valor m ${ }^{2}$ de construcción estandarizado & -1 & $-1,2$ & 0,0384 & $<0,0001$ \\
\hline Puntaje residual & $-0,3$ & $-1,1$ & 0,0066 & $<0,0001$ \\
\hline
\end{tabular}

Fuente: DANE (2011).

\subsubsection{MODElO DE ESTRATIFICACIÓN PARA El Distrito CAPITAL CON BASE EN EL AVALÚO CATASTRAL}

Como se mencionó anteriormente, una segunda posibilidad para implementar una nueva metodología de estratificación es considerar al Distrito Capital aparte del resto del país. En el capítulo 3, Bernal y Tejedor propusieron el diseño del valor unitario integral (VUI) como una variable adecuada para agrupar la información catastral, y, a lo largo del capítulo 4, se presentó evidencia respecto a que el vUI es la variable que mayor correlación tiene con indicadores socioeconómicos de bienestar social y capacidad de pago, además de condensar en un solo valor la información de entorno urbanístico, expresado por el valor del suelo, e información de características propias de la vivienda a través del valor de la construcción; estas dos características lo convierten en la variable más sobresaliente para estratificar los inmuebles residenciales en Bogotá, D.C. Adicionalmente, su definición tiene incorporadas las variables que podrían ser alternativas (explícitamente el valor de $\mathrm{m}^{2}$ del terreno o construcción e implícitamente el puntaje de la ficha catastral).

El procedimiento en este caso consiste en aplicar el método de K-Means sobre el valor unitario integral, buscando la clasificación de las unidades, en 
este caso los predios, en seis grupos homogéneos en el interior, en términos de dicha variable de clasificación. El método K-Means busca dividir M puntos de $\mathrm{N}$ dimensiones en $\mathrm{K}$ grupos, de forma que se minimice la suma de cuadrados en el interior de dichos grupos (Hartigan y Wong, 1979). En este caso, $\mathrm{N}=$ 1 , siendo la dimensión el vUI'

El vui busca captar la información del avalúo, eliminando los efectos del tamaño de predio, de adopción en el valor de terreno y construcción, y el efecto del estrato socioeconómico vigente en la conformación del avalúo. Para predios en propiedad horizontal (PH), el vUI es igual al avalúo del predio sobre el área construida; para predios que no son de propiedad horizontal (no $\mathrm{PH})$, el vUI es igual a la sumatoria del valor de metro cuadrado del terreno y de la construcción residencial?

El primer modelo en esta perspectiva parte del marco normativo legal vigente (L. 142/94), el cual limita el número de estratos hasta seis. Un segundo modelo contempla la posibilidad de no predeterminar los estratos, definiendo el número óptimo en busca de una mínima varianza posible dentro de los grupos. Esto llevaría a una definición más precisa de estrato, al tener grupos más homogéneos en el interior. Para obtener el número óptimo de grupos, se parte de una clasificación jerárquica, utilizando el método de Ward ${ }^{8}$. Por medio de este método, es posible determinar en cada paso del proceso iterativo una medida de la reducción de la varianza alcanzada. El número óptimo de grupos se elige cuando, al incluir un grupo adicional (estrato posible), la reducción de la varianza en el interior de los grupos no es estadísticamente significativa.

6 El algoritmo más implementado para la aplicación del método cumple los siguientes pasos:

a) se elige $\mathrm{K}$ centroides de forma aleatoria dentro del conjunto de $\mathrm{M}$ puntos; $\mathrm{b}$ ) se asigna cada punto al grupo que tiene la menor distancia euclidiana a su centroide; c) se recalculan los centroides de los K grupos como el promedio de los puntos que lo componen; d) nuevamente se calculan las distancias entre los puntos y cada uno de los K centroides recalculados, reubicando los puntos en el grupo con el centroide más cercano; e) se repiten los pasos hasta que el proceso converja, es decir, hasta que no se observen cambios en los grupos conformados.

7 Para el cálculo del VM2 de construcción residencial, se realizó el promedio, ponderado por el área, de los valores unitarios de las unidades de construcción residenciales (usos 001 y 002), donde $n$ es el número de unidades de construcción residenciales del predio, ValorUnitarioConstrucción es el valor unitario que es resultado del modelo en no PH y ValorM2 Terreno es el valor unitario de terreno asociado al predio.

8 http://www.docentes.unal.edu.co/cepardot/docs/SimposiosEstadistica/MetEstMulInvSocialParte4. pdf 


\subsection{Resultados}

A continuación, se presentan los resultados de los modelos de estratificación propuestos en términos de la distribución de viviendas estratificadas en Bogotá, D.C., teniendo en cuenta la información catastral 2012. La figura 5.2 muestra un comparativo de los dos modelos que resultan en una estratificación de acuerdo con la normatividad vigente, que establece que el número de estratos no puede ser mayor a seis.

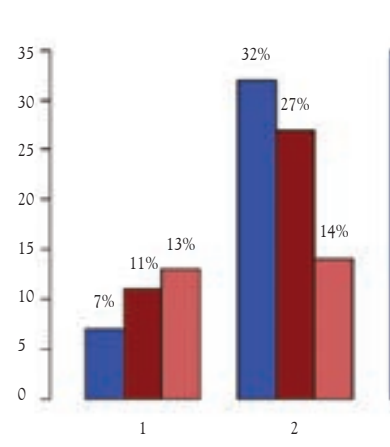

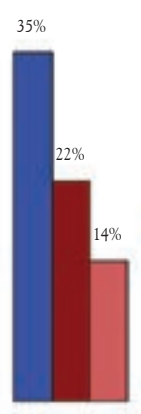

3

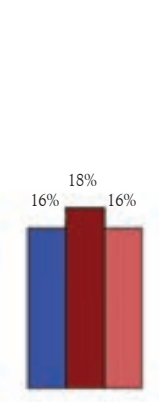

4

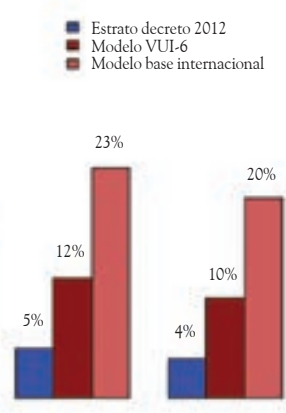

6

Figura 5.2. Distribución de predios según los escenarios en seis grupos

Fuente: elaboración de los autores con base en la EMB 2011.

Varios elementos se destacan tanto en el modelo de estratificación dentro de un marco nacional como en el que estudia a Bogotá de manera aislada (VUI). Los estratos 1,5 y 6 crecen significativamente respecto a la estratificación actual, mientras que los estratos 2 y 3 disminuyen de una manera drástica. El estrato 4 no varía radicalmente y agrupa un porcentaje similar de viviendas en los distintos modelos. Es importante resaltar que estas variaciones en la distribución de estratos recogen el cambio en el método de estratificación, así como una mejora efectiva en las condiciones de las viviendas, no registradas en la estratificación vigente.

Ahora, a pesar de que los cambios en la participación de cada estrato (respecto a la estratificación vigente) van en la misma dirección para ambos modelos, la magnitud es sustancialmente distinta, resultando en formas de distribución muy diferentes para cada propuesta. Bajo la perspectiva nacional, la distribución de estratos tiene un ligero sesgo a la izquierda (sesgo negativo), 
que lleva a que la contribución de cada estrato en el universo de viviendas del Distrito Capital aumente gradual, aunque muy leventemente, a medida que el estrato es mayor. La media de la contribución vigente por estrato es 15,8 puntos porcentuales, con una desviación de 13,2 pp, mientras que, para el modelo nacional, el promedio aumenta poco (a 16,6 pp), pero la desviación estándar se reduce a 4 pp. Esto implica, bajo el nuevo modelo, una distribución mucho más homogénea en cuanto al tamaño de los estratos.

La transición al segundo modelo (valor unitario integral) es menos drástica y la variación de la participación por estrato es menor para cada estrato. Esto resulta en una distribución sesgada a la derecha, con un promedio de participación de estrato igual a la propuesta nacional (16,6 pp), pero una desviación estándar de 6,7 pp.

Comparando las alternativas respecto al esquema vigente, los estratos bajos ( 1 y 2 ) disminuyen en 11 pp para el modelo nacional, mientras que no tienen ningún cambio para el modelo del vUi. Los estratos medios (3 y 4) caen en ambas propuestas, pero mucho más significativamente para el modelo nacional (18 pp frente a una caída de 8 pp en el modelo del vUI). De igual manera, el cambio de estratos altos $(5$ y 6 ) es mucho más pronunciado: el modelo nacional (en 34 pp) comparado con el vUI (13 pp).

La diferencia entre las distribuciones de las dos propuestas tiene explicación en la construcción de cada una. En el modelo nacional, la clasificación de Bogotá es relativa a todo el país, y, al ser la capital la ciudad con mayor desarrollo socioeconómico y urbanístico, resulta con una mayor clasificación de estratos altos comparado con la segunda alternativa que considera de manera independiente al Distrito Capital (modelo VUI).

Transitar hacia uno u otro modelo tiene implicaciones importantes. El modelo nacional concibe un sistema de pagos de SPD solidario entre todos los centros urbanos de Colombia, lo que en principio llevaría a pensar que este debe ser el referente final. Sin embargo, hay varios elementos que dificultan la transición hacia este modelo. Por un lado, la preconcepción del significado de estrato y el impacto de eventuales cambios de estrato en los hogares está tan arraigada en la ciudadanía que alteraciones muy bruscas en la estratificación llevaría a ser prácticamente inviable la implementación del nuevo esquema. Adicionalmente, la metodología de esta propuesta, que contiene diferentes pasos metodológicos complejos, impone un reto adicional para lograr una pedagogía adecuada que explique efectivamente las razones 
del eventual cambio de estrato de los distintos predios. Este punto se ilustra en más detalle al analizar las diversas matrices de transición de estrato entre modelos en la próxima sección.

Las fuertes variaciones entre composición de estratos y la complejidad metodológica del modelo base nacional están acompañadas por el hecho de que esta opción requiere un plan de implementación nacional que necesita de la alineación técnica y tecnológica por parte de los distintos catastros (con diagnósticos muy diferentes respecto a la actualización catastral y calidad y nivel de sistematización de la información).

El segundo modelo, por su parte, tiene ciertas ventajas. El Catastro Distrital presenta un avance claro en términos de actualización y sistematización frente al resto del país. Esto, junto con su complejidad urbanística y socioeconómica relativa, le permite plantear la posibilidad de avances alternativos para la ciudad, de diferentes herramientas de política pública como la estratificación, que pueden incluso ser referentes de hacia dónde se debe mover la herramienta a futuro para el resto del país. El resultado del modelo presenta una transición menos traumática con relación al primer modelo, respecto a cambios muy pronunciados en la distribución de estratos de la ciudad. Una tercera ventaja de esta propuesta es que es una estratificación que surge a partir de una clasificación de bienes inmuebles basada en una única variable, el valor único integral, lo que lleva a tener un proceso mucho más transparente y fácil de explicar a la ciudadanía.

La tercera alternativa considera, como se mencionó en la sección anterior, la posibilidad de que el número de estratos no esté definido exógenamente (en seis), sino que resulte de la composición y complejidad de los bienes inmuebles por estratificar, es decir que se determine endógenamente. En la medida en que agregar estratos nuevos reduzca la varianza promedio dentro de cada uno de los estratos, se llega a estratos con bienes inmuebles mucho más homogéneos dentro de sí.

El método de Ward es un proceso iterativo que estima la varianza interna promedio de grupos diferenciados (estratos) y determina la significancia del cambio de varianza a medida que nuevos estratos se van creando. En esta oportunidad, el número óptimo de estratos para la vigencia catastral 2012 es de nueve'.

9 La suma de cuadrados total (SCT) se puede descomponer en suma de cuadrados intragrupos (SCIn) y suma de cuadrados intergrupos (SCI); si se tiene un único grupo, la SCIn = SCT y, si cada indivi- 
El resultado final es entonces una clasificación con un promedio de participación porcentual por estrato de 11 pp con una desviación estándar de 5 pp (figura 5.3). El estrato de mayor tamaño es el tres, con un 19\% de los bienes inmuebles clasificados. Los estratos extremos son los de menor tamaño (estrato 1 y 2 , con 5 y $8 \%$, y estratos 8 y 9 , con 6 y $8 \%$ ).

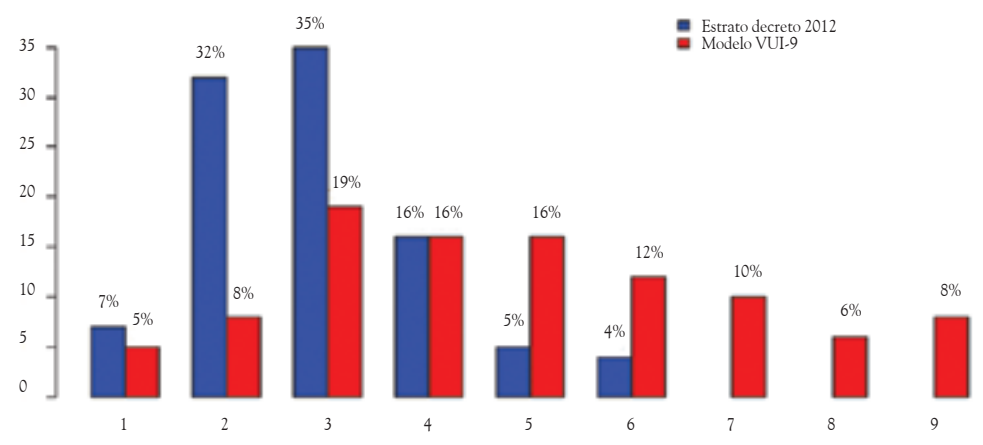

Figura 5.3. Clasificación para nueve grupos del valor unitario integral

Fuente: elaboración de los autores con base en la EMB 2011.

\subsubsection{IMPACTO SOCIAL Y ECONÓMICO DE ESCENARIOS}

\section{ALTERNATIVOS A LA ESTRATIFICACIÓN ACTUAL}

Más allá de analizar la viabilidad de los diferentes modelos a partir del cambio en la distribución de estratos, es fundamental entender en mayor detalle los eventuales impactos que el Distrito Capital acarrearía una vez implementados en la ciudad. Esta sección evalúa las consecuencias desde varios puntos de vista: transición específica de estratos actuales a estratos nuevos, y entre estratos subsidiables a contribuyentes; errores de inclusión y exclusión a través de varios referentes (probabilidad de estar en un estrato, dado un decil

duo es un grupo, la SCT $=$ SCI. En ese rango de posibles conformaciones de grupos, se puede escoger aquella que busque maximizar la SCIn (grupos homogéneos) sin llegar a considerar los individuos como grupos. Para este ejercicio, el punto de inflexión se encontró en nueve grupos. Al comparar el modelo de seis estratos con el de nueve, para el primero, el $44 \%$ de la suma de cuadrados total (SCT) se explica por la suma de cuadrados en el interior de los grupos (SCI), mientras que, para la clasificación en nueve grupos, se llega a un $52 \%$. Vale mencionar que SCT = SCI + SCE y el porcentaje que se calcula es SCI/SCT. 
de gasto, línea de pobreza, pobreza multidimensional y capacidad de pago); impacto financiero por medio de un cálculo general de incrementos o reducción de facturas.

\subsubsection{Impacto social}

Una aproximación al impacto social que pueda tener cada una de las propuestas de estratificación apunta a medir la movilidad que se genera, en términos de la migración de inmuebles residenciales entre la estratificación actual y cada una de las propuestas.

Los impactos son medidos bajo el supuesto de que el estrato neutro continúa siendo el 4 (por definición legal), que sería equivalente al grupo 4 de las clasificaciones planteadas. En el caso de la clasificación en nueve grupos, se requiere determinar un grupo neutro. Es decir, aquel donde no se aplican subsidios ni contribuciones, pagando así el costo de prestación del servicio público domiciliario.

\subsubsection{Transición entre estratos}

Las tablas 5.1.B a 5.3.A muestran la transición de predios entre el estrato actual y los diferentes estratos en cada uno de los modelos propuestos (base nacional, vUi y estratos endógenos). Adicionalmente, las 5.1.C y 5.2.B resumen la cantidad de inmuebles urbanos que cambian uno, dos, tres o más estratos.

Los resultados de esta transición van, por supuesto, en línea con los cambios generales de las distribuciones de estratos presentados en la sección anterior. Para el modelo VUI, se destaca que alrededor del 12\% de los predios residenciales aumenta dos o más estratos y que el 47,7\% de los predios no cambia de estrato. La mayor migración se observa en el estrato 5, donde solamente el 35,3\% de los predios permanece en ese estrato.

El impacto social observado en la propuesta nacional es mayor, ya que en este caso solamente el $27,84 \%$ de los predios permanece en el mismo estrato. Adicionalmente, alrededor del $36 \%$ de los predios aumenta dos o más estratos. El estrato donde mayor migración se observa es el 4, en el cual solamente permanece el 0,3\% de los predios. Bajo este escenario, en los estratos de los extremos (el 1 y el 6), se mantiene más porcentaje de predios en el mismo estrato. 
Tabla 5.1.B. Transición entre la estratificación vigente y el modelo base nacional

\begin{tabular}{|c|c|c|c|c|c|c|c|c|}
\hline Estrato actual & 1 & 2 & 3 & 4 & 5 & 6 & Total & $\%$ \\
\hline 1 & 94.536 & 7.213 & 549 & 184 & 3 & 0 & \multirow{2}{*}{102.485} & \multirow{2}{*}{$7,4 \%$} \\
\hline$\%$ & $92,2 \%$ & $7,0 \%$ & $0,5 \%$ & $0,2 \%$ & $0,0 \%$ & $0,0 \%$ & & \\
\hline 2 & 93.317 & 166.976 & 118.793 & 91.081 & 14.383 & 122 & \multirow{2}{*}{484.672} & \multirow{2}{*}{$32,5 \%$} \\
\hline$\%$ & $19,3 \%$ & $34,5 \%$ & $24,5 \%$ & $18,8 \%$ & $3,0 \%$ & $0,0 \%$ & & \\
\hline 3 & 1.568 & 29.647 & 76.580 & 141.839 & 249.452 & 18.481 & \multirow{2}{*}{517.567} & \multirow{2}{*}{$34,9 \%$} \\
\hline$\%$ & $0,3 \%$ & $5,7 \%$ & $14,8 \%$ & $27,4 \%$ & $48,2 \%$ & $3,6 \%$ & & \\
\hline 4 & 41 & 22 & 195 & 643 & 75.003 & 160.215 & \multirow{2}{*}{236.119} & \multirow{2}{*}{$15,9 \%$} \\
\hline$\%$ & $0,0 \%$ & $0,0 \%$ & $0,1 \%$ & $0,3 \%$ & $31,8 \%$ & $67,9 \%$ & & \\
\hline 5 & 9 & 19 & 27 & 104 & 6.628 & 68.832 & \multirow{2}{*}{75.619} & \multirow{2}{*}{$5,1 \%$} \\
\hline$\%$ & $0,0 \%$ & $0,0 \%$ & $0,0 \%$ & $0,1 \%$ & $8,8 \%$ & $91,0 \%$ & & \\
\hline 6 & 49 & 41 & 41 & 82 & 1.418 & 60.672 & \multirow{2}{*}{62.303} & \multirow{2}{*}{$4,3 \%$} \\
\hline$\%$ & $0,1 \%$ & $0,1 \%$ & $0,1 \%$ & $0,1 \%$ & $2,3 \%$ & $97,4 \%$ & & \\
\hline Total & 189.520 & 203.918 & 196.185 & 233.933 & 346.887 & 308.322 & \multirow{2}{*}{1.478 .765} & \\
\hline$\%$ & $12,8 \%$ & $13,8 \%$ & $13,3 \%$ & $15,8 \%$ & $23,5 \%$ & $20,8 \%$ & & \\
\hline
\end{tabular}

Fuente: elaboración de los autores con base en la EMB 2011.

Tabla 5.1.C. Resumen transición entre estratificación vigente y modelo base nacional

\begin{tabular}{l|c|c|c|c}
\hline \multirow{2}{*}{\multicolumn{1}{c}{ Tipo de cambio }} & \multicolumn{2}{c}{ Suben } & \multicolumn{2}{c}{ Bajan } \\
\cline { 2 - 5 } & Predios & $\%$ & Predios & $\%$ \\
\hline Cambian un estrato & 411.680 & $27.84 \%$ & 124.681 & $8,43 \%$ \\
\hline Cambian dos estratos & 501.297 & $33.90 \%$ & 1.699 & $0,11 \%$ \\
\hline Cambian tres o más estratos & 33.173 & $2.24 \%$ & 200 & $0,01 \%$ \\
\hline Total & 946.150 & $64.0 \%$ & 126.580 & $8,6 \%$ \\
\hline No cambian & & \multicolumn{2}{c}{$27,46 \%$} & \\
\hline
\end{tabular}

Fuente: elaboración de los autores con base en la EMB 2011. 
Modelos de estratificación socioeconómica a partir de la información catastral para la ciudad de Bogotá, D.C.

Tabla 5.2.A. Transición entre estratificación vigente y modelo por valor integral en seis grupos

\begin{tabular}{|c|c|c|c|c|c|c|c|c|}
\hline Estrato actual & 1 & 2 & 3 & 4 & 5 & 6 & Total & $\%$ \\
\hline 1 & 70.466 & 21.003 & 1.450 & 344 & 58 & 0 & \multirow{2}{*}{93.321} & \multirow{2}{*}{$7,4 \%$} \\
\hline$\%$ & $75,5 \%$ & $22,5 \%$ & $1,6 \%$ & $0,4 \%$ & $0,1 \%$ & $0,0 \%$ & & \\
\hline 2 & 72.763 & 259.013 & 85.855 & 42.642 & 18.823 & 321 & \multirow{2}{*}{479.417} & \multirow{2}{*}{$32,5 \%$} \\
\hline$\%$ & $15,2 \%$ & $54,0 \%$ & $17,9 \%$ & $8,9 \%$ & $3,9 \%$ & $0,1 \%$ & & \\
\hline 3 & 11.045 & 117.943 & 202.596 & 108.571 & 56.397 & 20.777 & \multirow{2}{*}{517.329} & \multirow{2}{*}{$34,9 \%$} \\
\hline$\%$ & $2,1 \%$ & $22,8 \%$ & $39,2 \%$ & $21,0 \%$ & $10,9 \%$ & $4,0 \%$ & & \\
\hline 4 & 949 & 3.479 & 38.314 & 90.382 & 63.670 & 39.764 & \multirow{2}{*}{236.558} & \multirow{2}{*}{$15,9 \%$} \\
\hline$\%$ & $0,4 \%$ & $1,5 \%$ & $16,2 \%$ & $38,2 \%$ & $26,9 \%$ & $16,8 \%$ & & \\
\hline 5 & 486 & 155 & 1.688 & 12.305 & 26.704 & 34.355 & \multirow{2}{*}{75.693} & \multirow{2}{*}{$5,1 \%$} \\
\hline$\%$ & $0,6 \%$ & $0,2 \%$ & $2,2 \%$ & $16,3 \%$ & $35,3 \%$ & $45,4 \%$ & & \\
\hline 6 & 317 & 323 & 395 & 3.077 & 9.219 & 49.750 & \multirow{2}{*}{63.081} & \multirow{2}{*}{$4,3 \%$} \\
\hline$\%$ & $0,5 \%$ & $0,5 \%$ & $0,6 \%$ & $4,9 \%$ & $14,6 \%$ & $78,9 \%$ & & \\
\hline Total & 156.026 & 401.916 & 330.298 & 257.321 & 174.871 & 144.967 & \multirow{2}{*}{1.465 .399} & \\
\hline$\%$ & $13,5 \%$ & $20,9 \%$ & $25,4 \%$ & $17,9 \%$ & $14,1 \%$ & $8,2 \%$ & & \\
\hline
\end{tabular}

Fuente: elaboración de los autores con base en la EMB 2011.

Tabla 5.2.B. Resumen transición entre estratificación vigente y modelo por valor integral en seis grupos

\begin{tabular}{l|c|c|c|c}
\hline \multirow{2}{*}{\multicolumn{1}{c}{ Tipo de cambio }} & \multicolumn{2}{c}{ Suben } & \multicolumn{2}{c}{ Bajan } \\
\cline { 2 - 5 } & Predios & $\%$ & Predios & $\%$ \\
\hline Cambian un estrato & 313.454 & $21,39 \%$ & 250.544 & $17,10 \%$ \\
\hline Cambian dos estratos & 140.253 & $9,57 \%$ & 19.289 & $1,32 \%$ \\
\hline Cambian tres o más estratos & 40.323 & $2,75 \%$ & 2.625 & $0,18 \%$ \\
\hline Total & 494.030 & $33,7 \%$ & 272.458 & $18,6 \%$ \\
\hline No cambian & & \multicolumn{2}{c}{$47,69 \%$} & \\
\hline
\end{tabular}

Fuente: elaboración de los autores con base en la EMB 2011. 


\subsubsection{UNA APROXIMACIÓN A LA DEFINICIÓN DE LOS GRUPOS SUBSIDIABLES Y CONTRIBUYENTES}

En un esquema de subsidios cruzados, determinar los grupos que son subsidiables y los que pueden ser objeto de contribución pasa por la definición de aquel grupo que, conforme a sus capacidades económicas no requeriría de subsidios ni al que se le podrían poner cargas adicionales, el denominado grupo 'neutro' ${ }^{10}$. Una forma para determinar el denominado grupo 'neutro' que se propuso en el trabajo del DANE (2011) ${ }^{11}$ recurre a la estimación del punto sobre la curva de Lorenz que tiene la misma pendiente a la curva de equidistribución (1:1). Conceptualmente, este punto identifica a la media de la capacidad de pago o ingreso de la población.

La figura 5.4 muestra la curva de Lorenz para la capacidad de pago, calculada con base en la información de la Encuesta multipropósito de Bogotá 2011. El grupo neutro, que, como se mencionó, no requeriría de subsidios ni contribuciones, se define como aquel que contiene el punto en el que la pendiente de la curva de Lorenz es la misma a la pendiente de la línea de equidistribución (45 grados), esto se representa por el punto negro en la gráfica y se define como capacidad de pago neutra (o capacidad de pago media). En la figura, se observa que este grupo neutro está contenido en el quinto estrato o grupo. Aquellos hogares que estén más alejados del punto neutro en la parte inferior de la distribución y que, en consecuencia, tienen una pendiente inferior a 1 son susceptibles de ser subsidiados, mientras aquellos que se encuentren por encima del punto neutro, con una pendiente mayor a 1, son llamados a contribuir ${ }^{12}$.

La magnitud del subsidio o la contribución podría determinarse en función de la proporción de la capacidad de pago de un grupo con relación a la capacidad de pago neutra. Una posibilidad es definir, utilizando una justificación de pobreza relativa como desigualdad, que aquellos hogares que se en-

10 En el esquema actual de subsidios y contribuciones, este grupo 'neutro' es el denominado estrato 4.

11 La propuesta fue incorporada en el trabajo del DANE por Paula Carolina Altamar R., Denis López y Alexis Maluendas.

12 Este mismo análisis se hizo para la clasificación en seis grupos del vUI, y el grupo neutro es el 4, igual a como se estableció en la Ley 142 de 1994. 
cuentran por debajo del $60 \%$ de la capacidad media son el grupo subsidiable. Este punto se ilustra en rojo ${ }^{13}$, el cual se ubica en el estrato/grupo 4.

En la tabla 5.3.A, se observa la transición de predios respecto a la estratificación vigente. En este caso, el impacto social puede verse mitigado por el cambio en el número de grupos, por lo que no se hace referencia a quienes suben o bajan de estrato. Se destaca que el estrato 1 migra mayormente a los grupos 1 y 2; el estrato 2, a los grupos 3 y 4 ; el estrato 3 , a los grupos 4 y 5 ; el estrato 4, a los grupos 6 y 7; el estrato 5, a los grupos 7, 8 y 9; y el estrato 6, a los grupos 8 y 9 .

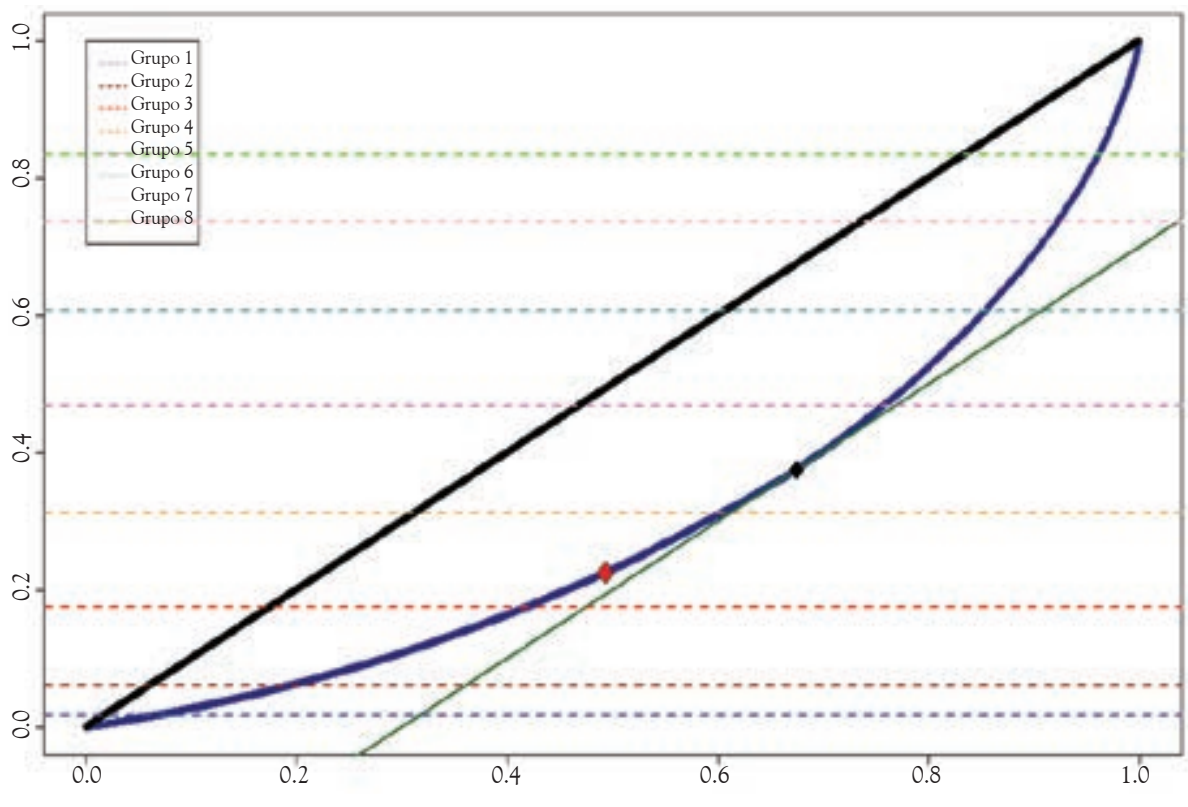

Figura 5.4. Curva de Lorenz para la capacidad de pago corriente y líneas de corte de la clasificación en nueve grupos por valor integral

Fuente: elaboración de los autores con base en la EMB 2011.

13 Las líneas de referencia por grupo se calculan como el porcentaje acumulado de la capacidad de pago por grupo. 
Tabla 5.3.A. Transición entre la estratificación vigente y la clasificación por valor integral en nueve grupos

\begin{tabular}{|c|c|c|c|c|c|c|c|c|c|c|c|}
\hline $\begin{array}{l}\text { Estrato } \\
\text { actual }\end{array}$ & 1 & 2 & 3 & 4 & 5 & 6 & 7 & 8 & 9 & Total & $\%$ \\
\hline 1 & 50.585 & 28.930 & 11.395 & 1.910 & 151 & 293 & 56 & 1 & 0 & \multirow{2}{*}{93.321} & \multirow{2}{*}{$7,4 \%$} \\
\hline$\%$ & $54,2 \%$ & $31,0 \%$ & $12,2 \%$ & $2,0 \%$ & $0,2 \%$ & $0,3 \%$ & $0,1 \%$ & $0,0 \%$ & $0,0 \%$ & & \\
\hline 2 & 18.888 & 85.108 & 193.930 & 92.850 & 43.023 & 27.521 & 15.934 & 2.123 & 40 & \multirow{2}{*}{479.417} & \multirow{2}{*}{$32,5 \%$} \\
\hline$\%$ & $3,9 \%$ & $17,8 \%$ & $40,5 \%$ & $19,4 \%$ & $9,0 \%$ & $5,7 \%$ & $3,3 \%$ & $0,4 \%$ & $0,0 \%$ & & \\
\hline 3 & 2.148 & 11.128 & 82.352 & 133.987 & 140.809 & 70.512 & 45.148 & 18.486 & 12.759 & \multirow{2}{*}{517.329} & \multirow{2}{*}{$34,9 \%$} \\
\hline$\%$ & $0,4 \%$ & $2,2 \%$ & $15,9 \%$ & $25,9 \%$ & $27,2 \%$ & $13,6 \%$ & $8,7 \%$ & $3,6 \%$ & $2,5 \%$ & & \\
\hline 4 & 482 & 488 & 1.202 & 12.095 & 40.931 & 66.536 & 52.505 & 34.348 & 27.971 & \multirow{2}{*}{236.558} & \multirow{2}{*}{$15,9 \%$} \\
\hline$\%$ & $0,2 \%$ & $0,2 \%$ & $0,5 \%$ & $5,1 \%$ & $17,3 \%$ & $28,1 \%$ & $22,2 \%$ & $14,5 \%$ & $11,8 \%$ & & \\
\hline 5 & 389 & 124 & 49 & 248 & 2.764 & 9.015 & 19.140 & 17.649 & 26.315 & \multirow{2}{*}{75.693} & \multirow{2}{*}{$5,1 \%$} \\
\hline$\%$ & $0,5 \%$ & $0,2 \%$ & $0,1 \%$ & $0,3 \%$ & $3,7 \%$ & $11,9 \%$ & $25,3 \%$ & $23,3 \%$ & $34,8 \%$ & & \\
\hline 6 & 82 & 276 & 194 & 256 & 385 & 2.529 & 4.274 & 10.643 & 44.442 & \multirow{2}{*}{63.081} & \multirow{2}{*}{$4,3 \%$} \\
\hline$\%$ & $0,1 \%$ & $0,4 \%$ & $0,3 \%$ & $0,4 \%$ & $0,6 \%$ & $4,0 \%$ & $6,8 \%$ & $16,9 \%$ & $70,5 \%$ & & \\
\hline Total & 72.574 & 126.054 & 289.122 & 241.346 & 228.063 & 176.406 & 137.057 & 83.250 & 111.527 & \multirow{2}{*}{1.465 .399} & \\
\hline$\%$ & $5,0 \%$ & $8,6 \%$ & $19,7 \%$ & $16,5 \%$ & $15,6 \%$ & $12,0 \%$ & $9,4 \%$ & $5,7 \%$ & $7,6 \%$ & & \\
\hline
\end{tabular}

Fuente: elaboración de los autores con base en la EMB 2011.

\subsubsection{Transición entre subsidiables y contribuyentes}

A continuación, se comparan los tres escenarios con relación a la estratificación vigente, en términos de la transición entre grupos potencialmente subsidiables, grupos contribuyentes y el grupo neutro, sabiendo que, en los escenarios de seis grupos, se supone como grupo neutro el estrato 4 , mientras que, en el escenario de nueve grupos, se toma como estrato neutro, de acuerdo con la sección previa, al grupo 5. Los resultados de transición se presentan en la tabla 5.3.B.

Los mayores cambios entre estratos subsidiables y contribuyentes se observan en la clasificación del modelo base nacional. Para este caso, prácticamente todos los hogares que hoy se encuentran en el estrato neutro o son contribuyentes pasan a contribuir en el nuevo esquema (99,6\% del estrato 
Tabla 5.3.B. Transición entre subsidiables y contribuyentes

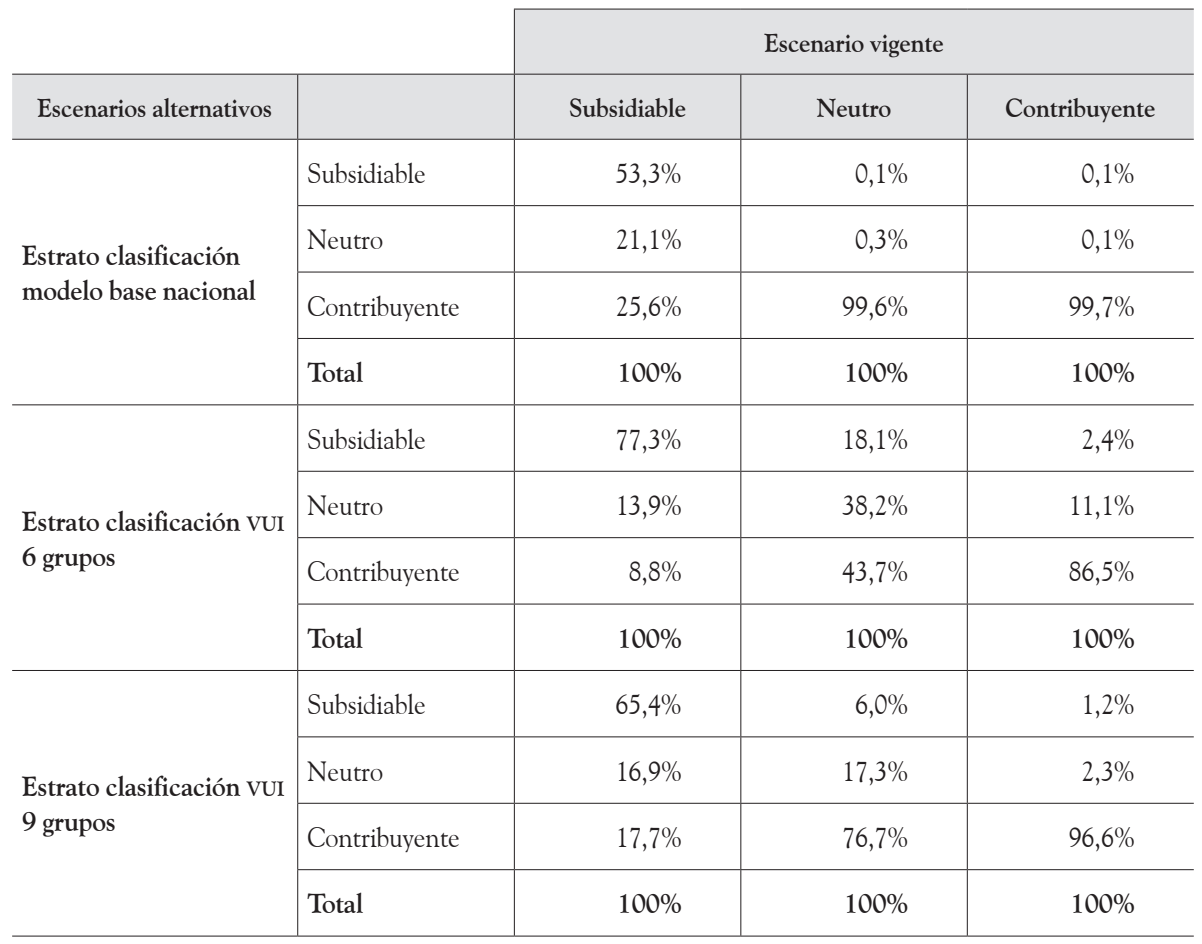

Fuente: elaboración de los autores con base en la EMB 2011.

neutro y 99,7\% de los contribuyentes actuales). Adicionalmente, de aquellos hogares que hoy reciben subsidios, el 53,3\% seguiría en el grupo subsidiable, el $21,1 \%$ pasaría a no recibir subsidio ni pagar contribuciones, mientras que el 25,6\% pasaría de recibir subsidios hoy en día a pagar una contribución.

De otro lado, el menor impacto social se observa en la clasificación directa sobre valor unitario en seis grupos, donde el 77,3\% de los predios subsidiables, el 38,2\% de los neutros y el 86,5\% de los contribuyentes no cambian de condición. En este caso, el 14\% de los hogares con subsidios actuales pasa al estrato neutro y el $9 \%$ entra ahora a contribuir, mientras que, de aquellos en estrato 4 hoy en día, el $44 \%$ pasaría a contribuir, mientras que el $18 \%$ recibiría subsidios en el nuevo esquema.

En términos de porcentajes de cambio en la condición del hogar, respecto a ser subsidiado o contribuyente, la propuesta de nueve estratos resulta en un punto intermedio entre los otros dos modelos alternativos. En este caso, el 
$96,6 \%$ de los contribuyentes siguen con esta condición, mientras que, para el estrato 4, el 76,7\% pasa a contribuir. Del grupo subsidiado actualmente, el $65,4 \%$ se mantiene recibiendo subsidios, mientras que el $18 \%$ pasa a contribuir.

Este resultado es un reflejo de la magnitud de la corrección de los errores de inclusión que presenta la estratificación vigente. Todos los modelos alternativos reducen los bienes inmuebles cuyos hogares recibirán subsidios y aumenta el número de contribuyentes.

\subsubsection{Impacto económico}

El impacto económico se mide a través del cálculo de los errores de exclusión e inclusión en la aplicación de subsidios y contribuciones en SPD, comparados con el estado de estos errores en la estratificación vigente. Los errores de exclusión e inclusión son medidos a partir de la información de los hogares de la Encuesta multipropósito de Bogotá 2011, definidos de la siguiente manera:

Error de exclusión: es el error en que se incurre cuando un hogar cumple los requerimientos para ser subsidiado en SPD, pero es clasificado dentro de los estratos o grupos neutros o contribuyentes. Este error tiende a ser autocorregible, puesto que es muy probable que hogares que habitan el inmueble mal clasificado soliciten la corrección.

Error de inclusión: es el error en que se incurre cuando un hogar que no cumple los requerimientos para ser subsidiado en SPD es clasificado dentro de los estratos o grupos subsidiables. Este error es poco probable que sea autocorregible, porque el hogar, aun teniendo la capacidad de pago para costear los servicios, no va a perder el beneficio de recibir un subsidio y pagar menos en SPD.

Para determinar los requerimientos de ser o no subsidiado, se recurre a varias aproximaciones, con el fin de determinar un panorama amplio del impacto económico. Los errores de exclusión se pueden medir de mejor forma evaluando la proporción de pobres (por línea de pobreza o por pobreza multidimensional) en los grupos neutros y contribuyentes, y la proporción de hogares sin capacidad de pago en estos mismos grupos. Los errores de inclusión se miden de mejor manera a través de la medición de las probabilidades de pertenecer a un grupo dado el nivel de ingresos. 


\subsubsection{Errores según probabilidad de pertenencia a un estrato dado el nivel de capacidad de pago}

Un esquema de estratificación socioeconómica tiene como objetivo clasificar adecuadamente aquellos inmuebles cuyos hogares requieren de subsidios en SPD y aquellos a cuyos hogares deben cobrárseles contribuciones. Un 'buen' diseño de estratificación llevaría a que, entre mayor ingreso o gasto tenga un hogar, mayor es la probabilidad de que el predio en que habita tenga asignado un estrato mayor.

Teniendo la probabilidad de pertenecer a un estrato, dado, por ejemplo, el decil de gasto del hogar, es decir:

$$
P[\text { estrato }=i \mid \text { decil de capacidad de pago }=j]
$$

resulta en $N$ distribuciones de probabilidad, siendo $N$ el número de estratos definidos (6 o 9).

Un esquema óptimo debe pretender que, a medida que aumenta el decil de capacidad de pago de un hogar, la probabilidad de pertenecer a un estrato más alto se incrementa y la probabilidad de pertenecer a un estrato más bajo disminuye. Por otro lado, para hogares en el decil 1, la probabilidad de estar en estrato 1 debería ser mayor a la de estar en el estrato 2, 3, 4, 5 y 6 (en ese orden). Así mismo, para los hogares con mayor capacidad de pago (decil 10), el estrato más probable debe ser el 6, seguido por el 5, 4, 3, 2 y 1 (en su orden).

La figura 5.5 presenta las probabilidades asociadas a la estratificación vigente, basadas en el índice de capacidad de pago, presentado en el capítulo 4. Allí se puede observar que, entre los deciles 1 a 9, es ampliamente probable pertenecer a los estratos 2 y 3 , que son subsidiables. Tener la gran mayoría de los hogares perteneciendo a los estratos 2 y 3 (cerca del 50\%), sin importar su nivel de capacidad de pago, alerta sobre los altos niveles en los errores de inclusión, pues solamente para el último decil de la capacidad de pago es más probable pertenecer a un estrato mayor. Esto permite entender mejor los fuertes cambios en las distribuciones (y la eventual disminución de los bienes inmuebles clasificados en estos dos estratos) cuando las comparamos con los modelos propuestos, en particular con el modelo base nacional.

Se destaca también que la probabilidad de pertenecer al estrato 1 nunca es mayor a la de pertenecer al 2, aun si se está en el primer decil de la capacidad de pago. De la misma forma, en el último decil es más probable ser de estrato 4 que de estrato 5 o 6. 


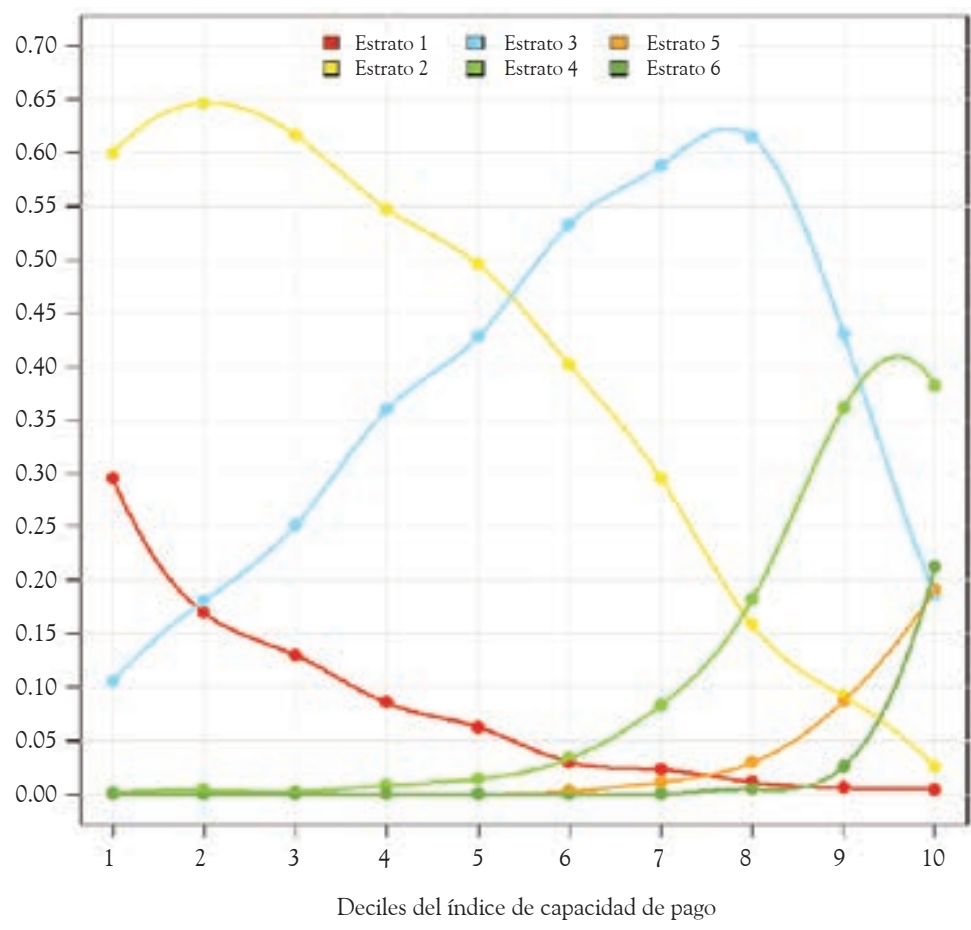

Figura 5.5. Probabilidad de estar clasificado en un estrato dado el decil de capacidad de pago en la estratificación vigente

Fuente: elaboración de los autores con base en la EMB 2011.

Las figuras 5.6 y 5.7 ilustran las diferentes distribuciones de probabilidad de pertenecer a un estrato determinado, dado el decil de gasto del hogar, para cada modelo alternativo propuesto.

Dentro de las diferentes opciones, la clasificación por modelo base nacional representa de mejor manera un comportamiento deseable de las probabilidades de pertenecer a un estrato. De forma general, se observa que los deciles bajos tienen mayor probabilidad de pertenecer a estratos bajos y los deciles altos, a estratos altos. En el primer decil, la probabilidad de pertenecer al grupo 1 es mayor que la de pertenecer al estrato 2, 3, 4, 5 y 6, en ese orden. En la misma línea, en el último decil tiene una probabilidad mayor de estar asignado al estrato 6 , seguidos en su orden la probabilidad de estar en estratos $5,4,3,2$ y 1 (estos últimos con una probabilidad muy cercana a cero). 
Adicionalmente, entre mayor es la capacidad de pago, la distribución de probabilidad que domina a las demás aumenta. Esto implica que, a medida que el decil aumenta, el estrato al que el hogar tiene mayor probabilidad de ubicarse se incrementa, lo cual es un resultado bastante positivo.

Respecto al modelo de clasificación directa (VUI) de seis estratos, el comportamiento de las probabilidades es similar al observado en la estratificación vigente (con predominancia de los estratos 2 y 3), pero menos acentuado. Así mismo, se mejora la probabilidad en el estrato 6, que se muestra mayor en el último decil, aunque se mantiene en el primer decil, que es más probable ser de estrato 2 que de estrato 1. Los resultados para la clasificación directa en nueve grupos son similares a los de la clasificación directa en seis grupos.

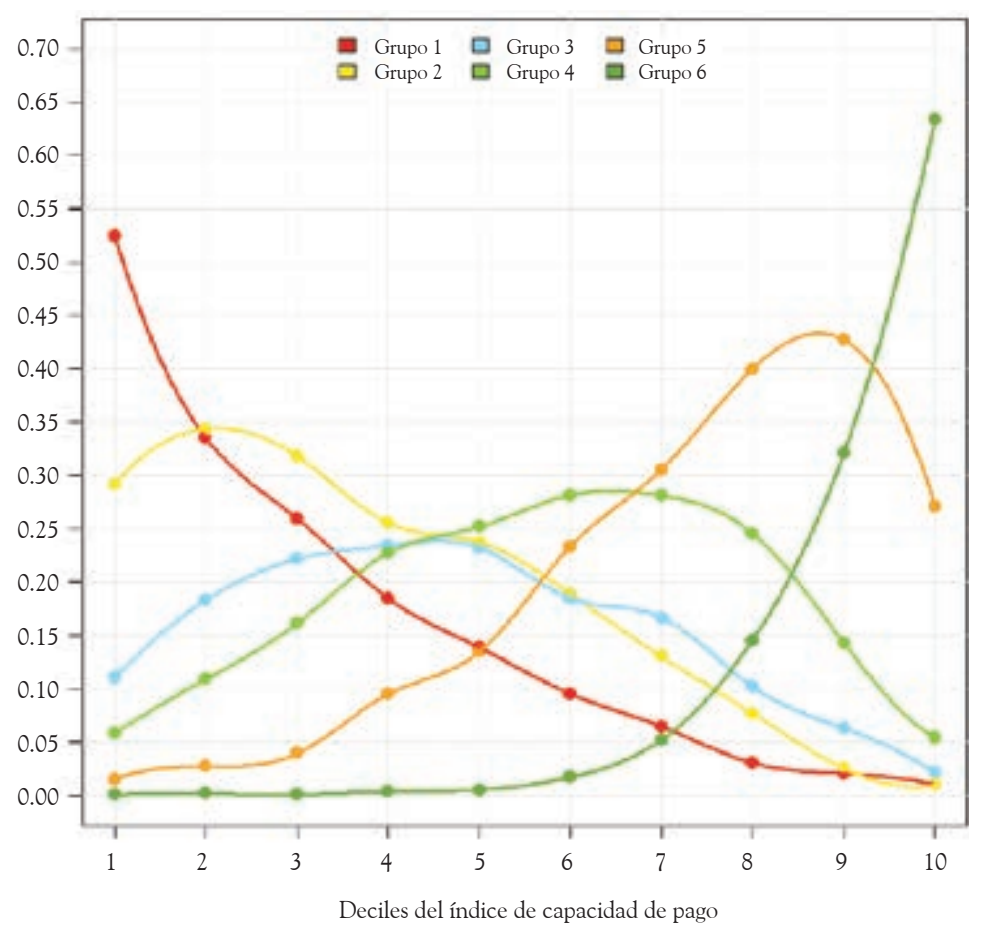

Figura 5.6. Probabilidad de estar clasificado en un estrato dado el decil de capacidad de pago según clasificación de modelos alternativos 


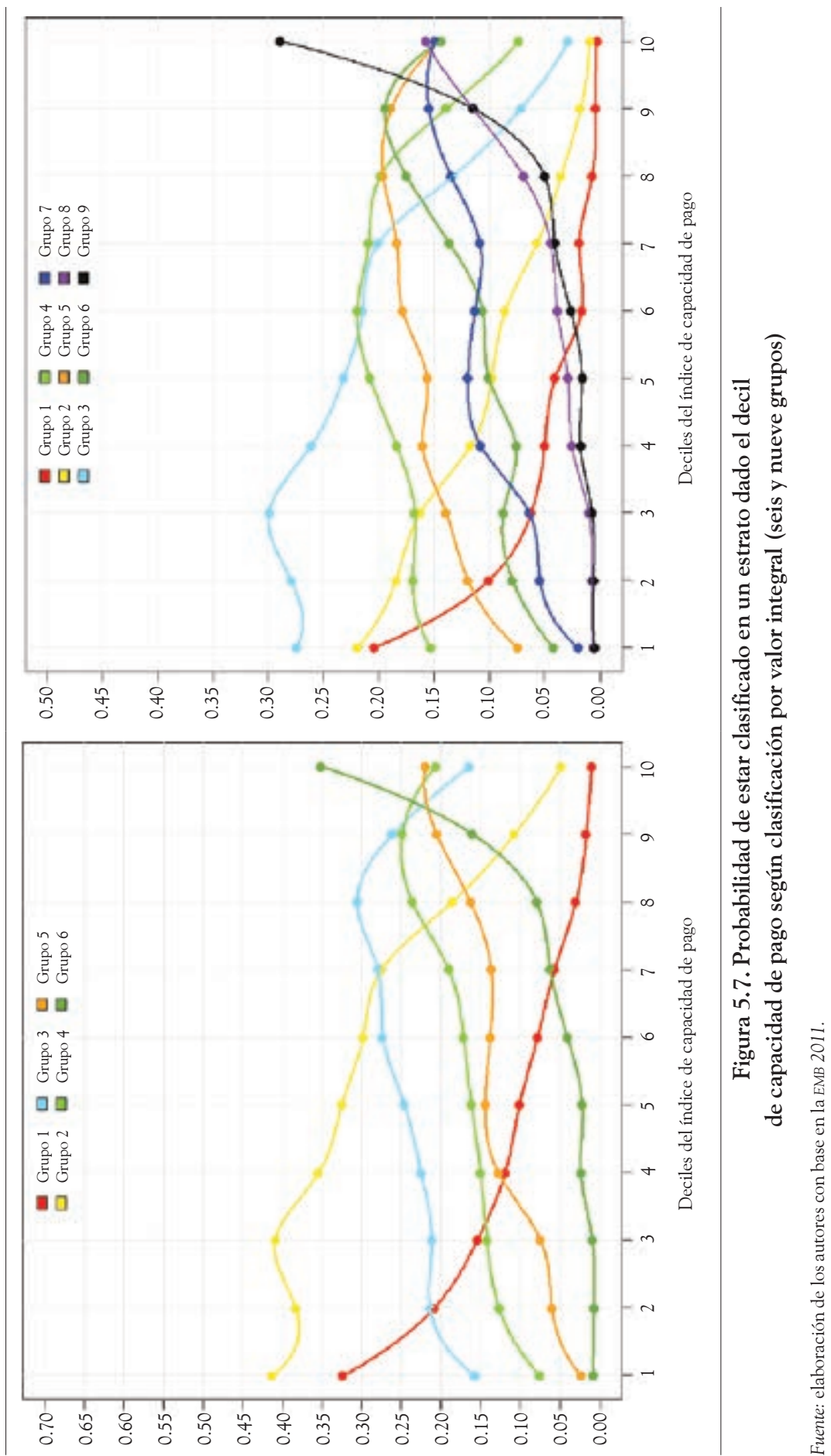




\subsubsection{Errores según línea de pobreza}

La línea de pobreza es un umbral que se define para determinar un nivel mínimo de ingresos a partir del cual un hogar o familia es considerado pobre. Para este análisis, se utilizó una línea de pobreza de 177.562 pesos per capita ${ }^{14}$. El objetivo es determinar los errores de exclusión e inclusión a partir del porcentaje de hogares pobres que se encuentran en los grupos.

En la tabla 5.4, se muestran los porcentajes de pobres entre los grupos de las clasificaciones comparados con los porcentajes de la estratificación vigente. Se resalta en color gris el estrato neutro en cada clasificación. Se puede observar que la estratificación vigente presenta errores de exclusión más pequeños con un $0,2 \%$ de los pobres ubicados en los estratos no subsidiables. Entre las clasificaciones propuestas, en línea con los ejercicios anteriores, la que reduce más los errores de exclusión es la resultante del modelo base nacional con el $8,7 \%$ de los hogares pobres ubicados en grupos no subsidiables. También, se destaca que esta clasificación con la propuesta base nacional permite ubicar más hogares pobres en el estrato 1 que las otras clasificaciones propuestas y que la estratificación actual.

En la tabla 5.5, se exponen los porcentajes de pobres en el interior de cada estrato. Esta medida permite identificar las clasificaciones que asignan más apropiadamente los pobres en los diferentes grupos. Se espera que los primeros grupos acumulen una mayor proporción de pobres, lo que reduciría el riesgo de encontrar errores de inclusión. La clasificación donde más pobres se ubican en el primer grupo es la directa en nueve grupos (51,3\%). También, se observan altos porcentajes de pobres en los grupos subsidiables, por lo que dicha clasificación puede presentar los menores errores de inclusión.

Mayores errores de exclusión de las propuestas alternativas tienen una explicación lógica. El proceso de estratificación socioeconómica actual lleva años implementándose, lo que permite ir corrigiendo gradualmente estas exclusiones (hogares en inmuebles mal clasificados en estratos contribuyentes reclaman para ser reclasificados en estratos bajos).

14 Se usa la línea de pobreza nacional con línea base ENIG 2006-2007, actualizada por IPC (DANE, Pobreza monetaria y multidimensional en Colombia, boletín de prensa, mayo de 2012). 
Tabla 5.4. Porcentaje de pobres entre grupos según línea de pobreza

\begin{tabular}{c|c|c|c|c}
\hline Estrato/grupo & $\begin{array}{c}\text { Estratificación } \\
\text { vigente }\end{array}$ & $\begin{array}{c}\text { Clasificación modelo } \\
\text { base nacional }\end{array}$ & $\begin{array}{c}\text { Clasificación vuI 6 } \\
\text { grupos }\end{array}$ & $\begin{array}{c}\text { Clasificación vUI 9 } \\
\text { grupos }\end{array}$ \\
\hline 1 & $16,2 \%$ & $18,7 \%$ & $31,0 \%$ & $10,4 \%$ \\
\hline 2 & $50,6 \%$ & $34,5 \%$ & $25,2 \%$ & $15,5 \%$ \\
\hline 3 & $25,4 \%$ & $20,4 \%$ & $15,2 \%$ & $24,6 \%$ \\
\hline 4 & $4,5 \%$ & $13,5 \%$ & $12,4 \%$ & $15,6 \%$ \\
\hline 5 & $1,6 \%$ & $8,1 \%$ & $9,8 \%$ & $12,5 \%$ \\
\hline 7 & $1,7 \%$ & $4,8 \%$ & $6,3 \%$ & $8,4 \%$ \\
\hline 8 & & & & $6,6 \%$ \\
\hline 9 & & & & $2,7 \%$ \\
\hline
\end{tabular}

Fuente: elaboración de los autores con base en la EMB 2011.

Tabla 5.5. Porcentaje de pobres dentro de los grupos según línea de pobreza

\begin{tabular}{c|c|c|c|c}
\hline Estrato/grupo & $\begin{array}{c}\text { Estratificación } \\
\text { vigente }\end{array}$ & $\begin{array}{c}\text { Clasificación modelo } \\
\text { base nacional }\end{array}$ & $\begin{array}{c}\text { Clasificación vUI 6 } \\
\text { grupos }\end{array}$ & $\begin{array}{c}\text { Clasificación vUI 9 } \\
\text { grupos }\end{array}$ \\
\hline 1 & $48,4 \%$ & $42,0 \%$ & $39,1 \%$ & $51,3 \%$ \\
\hline 2 & $23,2 \%$ & $23,6 \%$ & $20,5 \%$ & $31,3 \%$ \\
\hline 3 & $4,8 \%$ & $13,3 \%$ & $10,3 \%$ & $19,5 \%$ \\
\hline 4 & $0,3 \%$ & $5,6 \%$ & $7,9 \%$ & $12,9 \%$ \\
\hline 5 & $0,0 \%$ & $1,2 \%$ & $3,3 \%$ & $8,2 \%$ \\
\hline 7 & $0,0 \%$ & $0,1 \%$ & $1,4 \%$ & $6,4 \%$ \\
\hline 8 & & & & $3,7 \%$ \\
\hline 9 & & & & $1,4 \%$ \\
\hline
\end{tabular}

Fuente: elaboración de los autores con base en la EMB 2011.

\subsubsection{Errores según pobreza multidimensional}

El índice de pobreza multidimensional (IPM) es uno de los indicadores más novedosos en la medición de la pobreza en el mundo. Desarrollado por la Oxford Poverty \& Human Development Initiative (OPHI), identifica un conjunto de privaciones en las necesidades básicas de los hogares y determina la 
condición de pobreza a través de la suma ponderada de dichas privaciones. El indicador ha sido aplicado en Colombia por medio de la evaluación de quince privaciones y considerando un hogar como pobre si la suma ponderada de privaciones excede el $30 \%$. Para mayor detalle del modelo teórico, se puede ver Alkire y Foster (2009).

En la tabla 5.6, se presentan los porcentajes de pobres entre los grupos, donde se observan resultados similares a los señalados en los errores por línea de pobreza. Esto es, menores errores de exclusión en la estratificación vigente, seguida de la clasificación por modelo base nacional. Así mismo, el porcentaje de pobres dentro de los estratos muestra un mejor comportamiento en la clasificación directa en nueve grupos, como se puede observar de la tabla 5.7.

Tabla 5.6. Porcentaje de pobres entre grupos según índice de pobreza multidimensional

\begin{tabular}{c|c|c|c|c}
\hline Estrato/grupo & $\begin{array}{c}\text { Estratificación } \\
\text { vigente }\end{array}$ & $\begin{array}{c}\text { Clasificación modelo } \\
\text { base nacional }\end{array}$ & $\begin{array}{c}\text { Clasificación vUI 6 } \\
\text { grupos }\end{array}$ & $\begin{array}{c}\text { Clasificación VUI 9 } \\
\text { grupos }\end{array}$ \\
\hline 1 & $18,2 \%$ & $33,9 \%$ & $21,1 \%$ & $11,6 \%$ \\
\hline 2 & $53,5 \%$ & $26,0 \%$ & $34,2 \%$ & $17,2 \%$ \\
\hline 3 & $25,3 \%$ & $17,7 \%$ & $21,5 \%$ & $22,9 \%$ \\
\hline 4 & $2,7 \%$ & $13,3 \%$ & $13,9 \%$ & $17,3 \%$ \\
\hline 5 & $0,2 \%$ & $7,6 \%$ & $6,4 \%$ & $13,4 \%$ \\
\hline 7 & $0,1 \%$ & $1,6 \%$ & $2,9 \%$ & $8,3 \%$ \\
\hline 8 & & & & $5,8 \%$ \\
\hline 9 & & & & $1,4 \%$ \\
\hline
\end{tabular}

Fuente: elaboración de los autores con base en la EMB 2011.

Tabla 5.7. Porcentaje de pobres dentro de los grupos según índice de pobreza multidimensional

\begin{tabular}{c|c|c|c|c}
\hline Estrato/grupo & $\begin{array}{c}\text { Estratificación } \\
\text { vigente }\end{array}$ & $\begin{array}{c}\text { Clasificación modelo } \\
\text { base nacional }\end{array}$ & $\begin{array}{c}\text { Clasificación VUI 6 } \\
\text { grupos }\end{array}$ & $\begin{array}{c}\text { Clasificación vUI 9 } \\
\text { grupos }\end{array}$ \\
\hline 1 & $29,7 \%$ & $26,6 \%$ & $25,2 \%$ & $31,7 \%$ \\
\hline 2 & $17,5 \%$ & $17,7 \%$ & $14,9 \%$ & $21,7 \%$ \\
\hline 3 & $8,7 \%$ & $14,5 \%$ & $11,2 \%$ & $14,0 \%$ \\
\hline
\end{tabular}




\begin{tabular}{c|c|c|c|c}
\hline Estrato/grupo & $\begin{array}{c}\text { Estratificación } \\
\text { vigente }\end{array}$ & $\begin{array}{c}\text { Clasificación modelo } \\
\text { base nacional }\end{array}$ & $\begin{array}{c}\text { Clasificación vUI 6 } \\
\text { grupos }\end{array}$ & $\begin{array}{c}\text { Clasificación vUI 9 } \\
\text { grupos }\end{array}$ \\
\hline 4 & $3,0 \%$ & $9,3 \%$ & $10,0 \%$ & $12,4 \%$ \\
\hline 5 & $0,6 \%$ & $4,9 \%$ & $6,3 \%$ & $10,5 \%$ \\
\hline 6 & $0,7 \%$ & $1,6 \%$ & $4,8 \%$ & $9,0 \%$ \\
\hline 7 & & & & $7,0 \%$ \\
\hline 8 & & & & $3,5 \%$ \\
\hline 9 & & & & $4,4 \%$ \\
\hline
\end{tabular}

Fuente: elaboración de los autores con base en la EMB 2011.

\subsubsection{Errores según hogares sin capacidad de pago}

Se fundamenta en la identificación de hogares cuyos ingresos no alcanzan a cubrir su gasto mínimo en bienes básicos, mediante la utilización del enfoque de 'bienes básicos' y considerando los ordenamientos jerárquicos y lexicográficos de las necesidades. Un mayor detalle de la metodología se puede ver en CID (2012).

Como en el caso de las mediciones por pobreza, se observan menores errores de exclusión en la estratificación vigente, pero la clasificación del modelo base nacional acumula un mayor porcentaje de hogares sin capacidad de pago en el primer estrato (ver tabla 5.8).

De otro lado, el comportamiento de la proporción de hogares sin capacidad de pago dentro de los grupos es similar en todas las clasificaciones (tabla 5.9).

Tabla 5.8. Porcentaje de hogares sin capacidad de pago entre grupos

\begin{tabular}{c|c|c|c|c}
\hline Estrato/grupo & $\begin{array}{c}\text { Estratificación } \\
\text { vigente }\end{array}$ & $\begin{array}{c}\text { Clasificación modelo } \\
\text { base nacional }\end{array}$ & $\begin{array}{c}\text { Clasificación vUI 6 } \\
\text { grupos }\end{array}$ & $\begin{array}{c}\text { Clasificación vUI 9 } \\
\text { grupos }\end{array}$ \\
\hline 1 & $15,8 \%$ & $29,9 \%$ & $19,4 \%$ & $9,4 \%$ \\
\hline 2 & $49,6 \%$ & $24,6 \%$ & $36,2 \%$ & $16,7 \%$ \\
\hline 3 & $28,4 \%$ & $15,4 \%$ & $21,4 \%$ & $26,5 \%$ \\
\hline 4 & $4,4 \%$ & $14,5 \%$ & $11,8 \%$ & $17,1 \%$ \\
\hline 5 & $1,2 \%$ & $11,2 \%$ & $7,9 \%$ & $11,2 \%$ \\
\hline 6 & $0,6 \%$ & $4,4 \%$ & $3,4 \%$ & $7,9 \%$ \\
\hline
\end{tabular}


Modelos de estratificación socioeconómica a partir de la información catastral para la ciudad de Bogotá, D.C.

\begin{tabular}{c|c|c|c|c}
\hline Estrato/grupo & $\begin{array}{c}\text { Estratificación } \\
\text { vigente }\end{array}$ & $\begin{array}{c}\text { Clasificación modelo } \\
\text { base nacional }\end{array}$ & $\begin{array}{c}\text { Clasificación VUI 6 } \\
\text { grupos }\end{array}$ & $\begin{array}{c}\text { Clasificación VUI 9 } \\
\text { grupos }\end{array}$ \\
\hline 7 & & & & $5,9 \%$ \\
\hline 8 & & & & $3,0 \%$ \\
\hline 9 & & & & $2,4 \%$ \\
\hline
\end{tabular}

Fuente: elaboración de los autores con base en la EMB 2011.

Tabla 5.9. Porcentaje de hogares sin capacidad de pago dentro de los grupos

\begin{tabular}{c|c|c|c|c}
\hline Estrato/grupo & $\begin{array}{c}\text { Estratificación } \\
\text { vigente }\end{array}$ & $\begin{array}{c}\text { Clasificación modelo } \\
\text { base nacional }\end{array}$ & $\begin{array}{c}\text { Clasificación vUI 6 } \\
\text { grupos }\end{array}$ & $\begin{array}{c}\text { Clasificación vUI 9 } \\
\text { grupos }\end{array}$ \\
\hline 1 & $37,1 \%$ & $34,0 \%$ & $33,4 \%$ & $35,5 \%$ \\
\hline 2 & $24,2 \%$ & $24,8 \%$ & $24,2 \%$ & $31,9 \%$ \\
\hline 3 & $14,5 \%$ & $19,0 \%$ & $16,8 \%$ & $24,8 \%$ \\
\hline 4 & $7,7 \%$ & $14,9 \%$ & $12,7 \%$ & $18,4 \%$ \\
\hline 5 & $6,7 \%$ & $10,6 \%$ & $11,2 \%$ & $13,3 \%$ \\
\hline 6 & $4,7 \%$ & $6,8 \%$ & $8,0 \%$ & $12,6 \%$ \\
\hline 7 & & & & $10,4 \%$ \\
\hline 9 & & & & $7,5 \%$ \\
\hline
\end{tabular}

Fuente: elaboración de los autores con base en la EMB 2011.

\subsubsection{IMPACTO FINANCIERO}

Se propone aquí medir el impacto financiero a través de la evaluación del balance de subsidios y contribuciones en SPD, entre los escenarios evaluados. Sin embargo, dado que no es posible asimilar las clasificaciones propuestas a la información comercial de los servicios públicos domiciliarios, es necesario establecer algunas consideraciones:

1) La evaluación solo se realiza en los escenarios de seis grupos, ya tienen un régimen tarifario establecido, de acuerdo con la normatividad vigente. En el caso de la clasificación en nueve grupos, tendría que plantearse un régimen tarifario diferente, aspecto que compete a las respectivas comisiones de regulación, razón por la cual no se profundiza en este estudio. 
2) Se supone que el consumo medio en los inmuebles en cada grupo no cambia al ingresar o migrar nuevos inmuebles.

3) Se utiliza la información agregada de subsidios y contribuciones extraída de los reportes comerciales del Sistema Único de Información (SUI) de la Superintendencia de Servicios Públicos Domiciliarios (SSPD).

4) El ejercicio se efectúa para los servicios de acueducto, alcantarillado, aseo, energía y gas natural.

5) En el componente de subsidios, se suman solamente los valores reportados en los estratos 1, 2 y 3 . En el componente de contribuciones, se usan los valores reportados en los estratos 5 y 6 , y en los sectores: industrial, comercial, oficial, especial, temporal y multiusuario mixto.

6) Se supone que la relación facturas-predios es la misma dentro de todos los grupos generados por las clasificaciones.

El proceso consiste en determinar inicialmente, en cada estrato socioeconómico vigente, el subsidio o contribución promedio mes por factura. El monto total de subsidios/contribuciones para 2012 por estrato se divide en el número de facturas del año y luego entre doce para mensualizar el dato.

Posteriormente, se multiplica el porcentaje de predios de cada grupo en las dos clasificaciones evaluadas por el número total de facturas del año, con el fin de aproximarse al número de facturas dentro de cada grupo en las nuevas clasificaciones.

Finalmente, se multiplica el subsidio/contribución promedio mes por el número de facturas de cada grupo, obteniéndose así el valor del subsidio/ contribución total mes dentro de cada grupo. Al realizar la diferencia entre las contribuciones y subsidios, se obtiene el balance financiero que se pretende evaluar.

En las tablas 5.10 a 5.14, se evidencia el balance entre subsidios y contribuciones independientemente para cada SPD, frente a la situación del balance actual; a su vez, se supone que los porcentajes de subsidios y contribuciones se mantienen de acuerdo con la normatividad vigente. Antes se mostró que un número importante de inmuebles transitaría de estratos subsidiables a contribuyentes, por lo que es de esperarse que el balance del sistema mejore notablemente; como se observa en las tablas siguientes, se pasa de un sistema que para la ciudad de Bogotá, D.C., tiene más subsidios que contribuciones, saldo en rojo, a un sistema que aportaría al fondo de solidaridad nacional. 


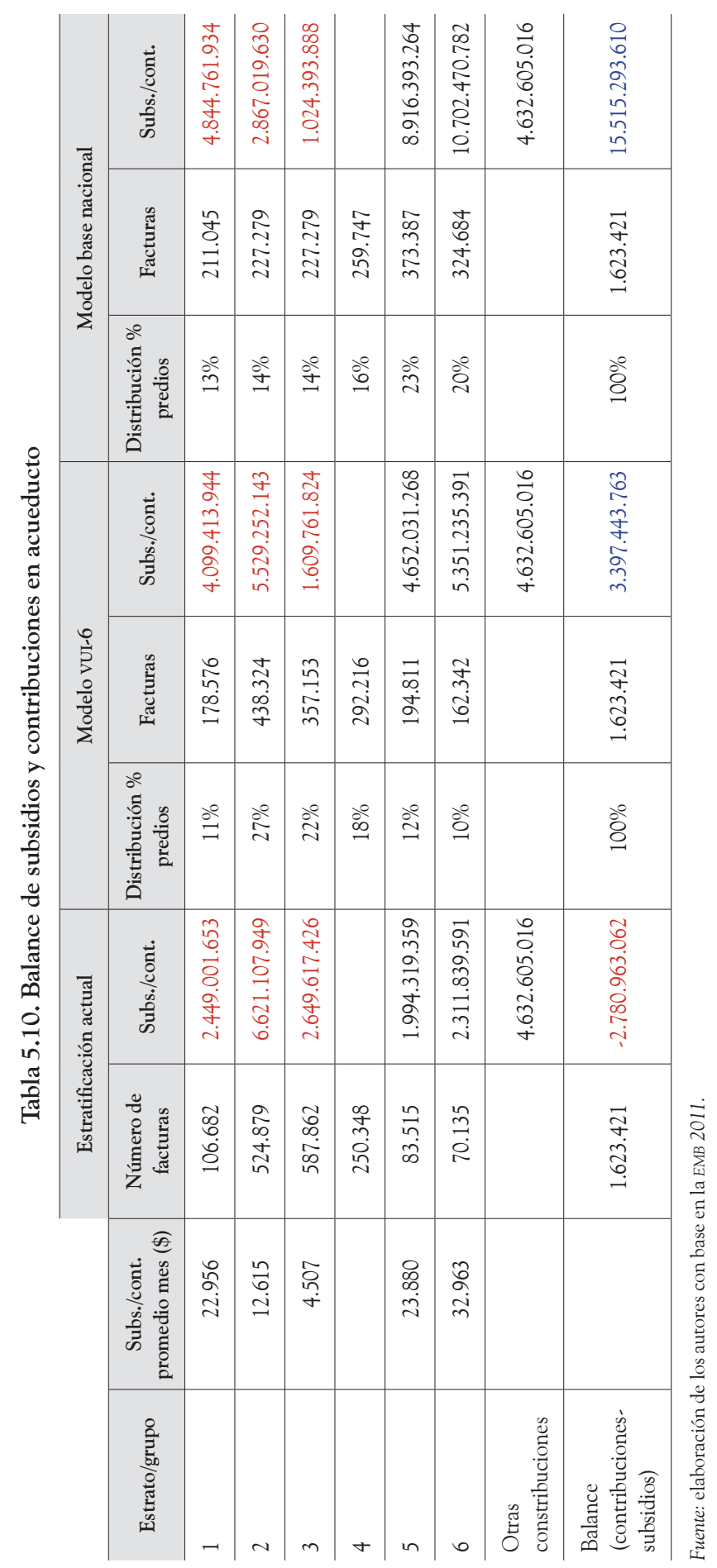




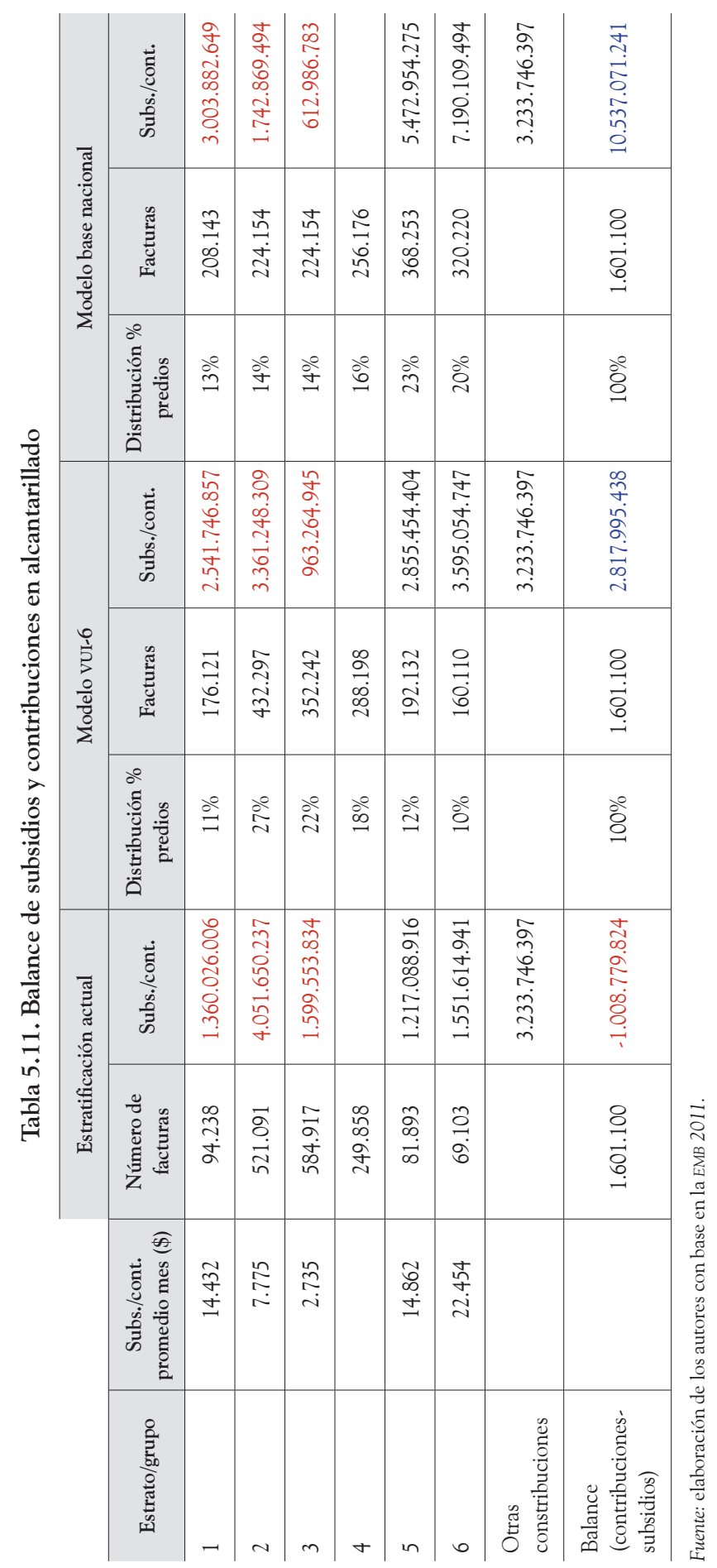




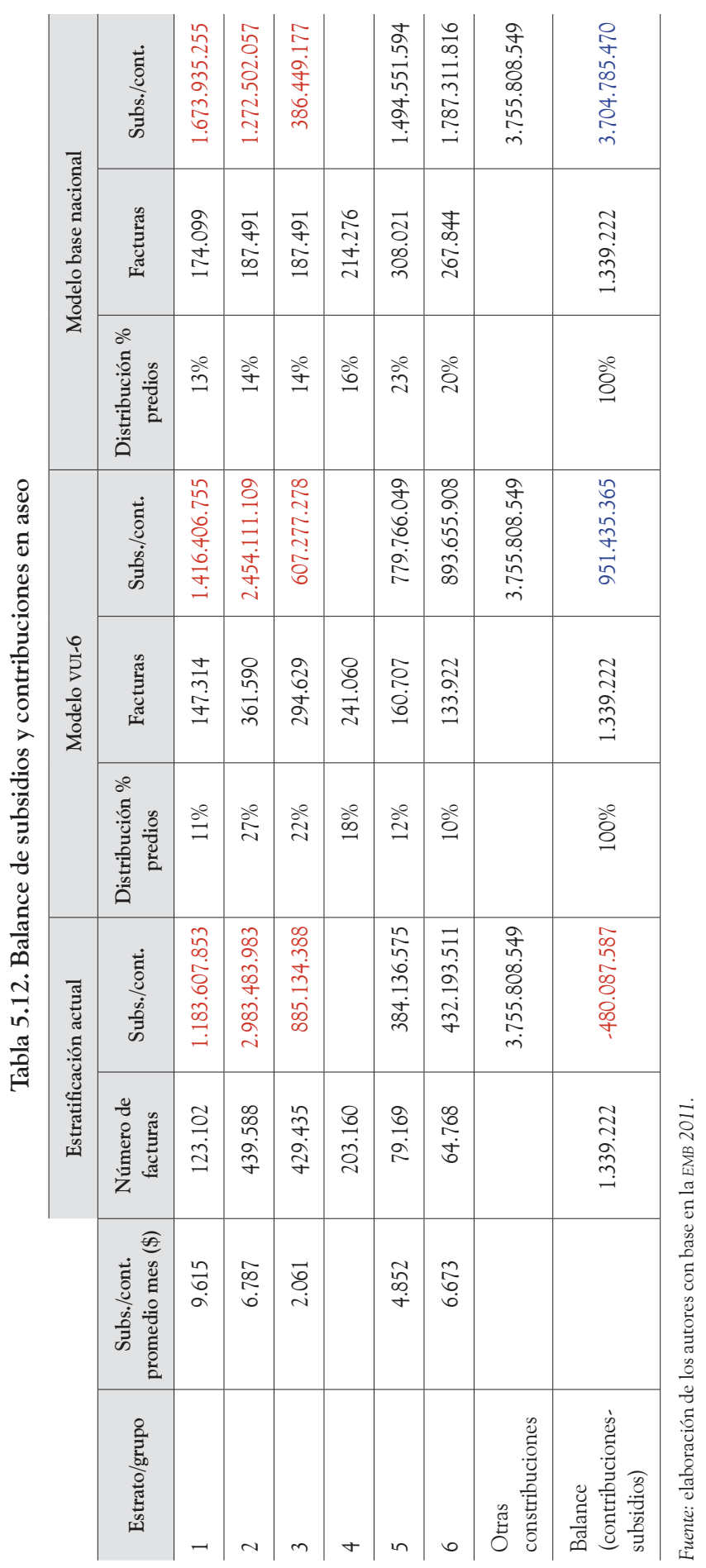




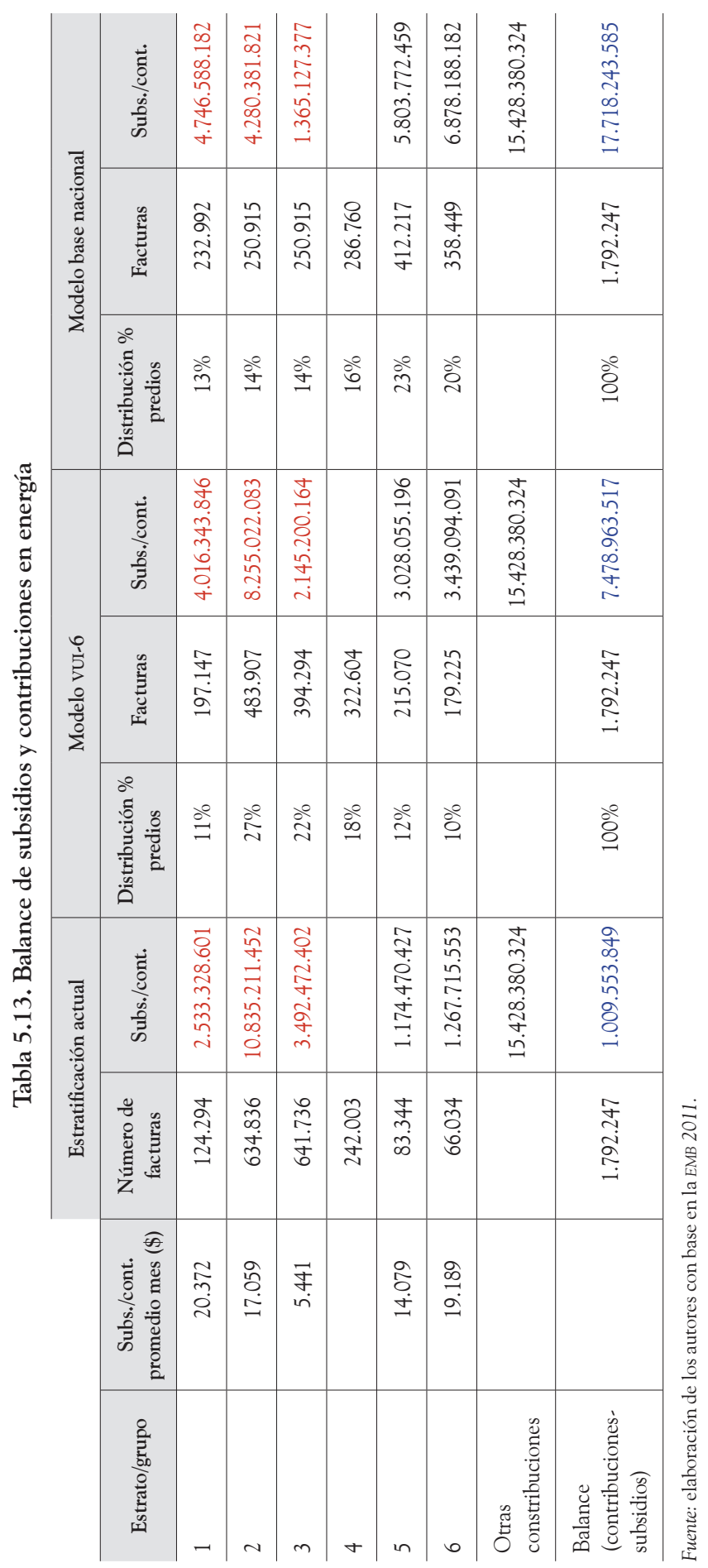




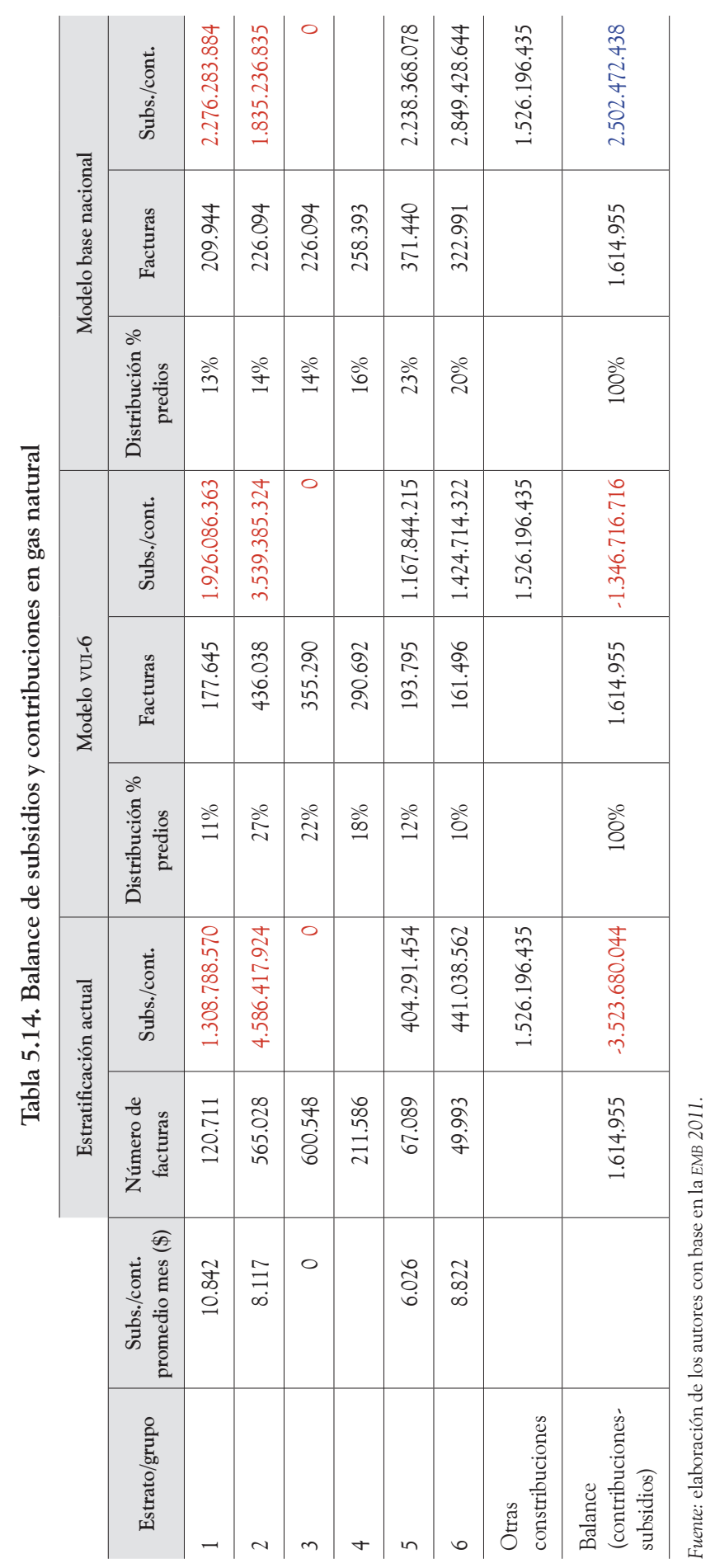


Comparando con el modelo que resulta en un cambio de composición de estratos menos drásticos (VUI), el balance entre subsidios y contribuciones pasa en acueducto de un saldo negativo de 2,7 miles de millones de pesos a un superávit de 3,4 miles de millones. El cambio más drástico se presenta en energía eléctrica, donde el balance pasa de un déficit de mil millones de pesos a un superávit de 7,5 miles de millones. Para el modelo base nacional, estos cambios se agrandan considerablemente.

Las estimaciones del balance financiero bajo los nuevos modelos liberan de manera importante recursos públicos y permiten pensar en la posibilidad de aumentar subsidios a los más pobres o en la definición de instrumentos complementarios de apoyo para este grupo.

\section{Conclusiones}

Los resultados de capítulo llevan a una reflexión de fondo respecto al sistema de subsidios de SPD establecido en Colombia. A pesar de que una eventual estratificación socioeconómica basada en información catastral presenta una mejora notable en los errores de inclusión, aún no refleja un escenario óptimo que diferencie adecuadamente hogares subsidiables de contribuyentes. Esto lleva a plantear la posibilidad de explorar esquemas alternativos para el sistema subsidiario de pago de SPD, diferentes a la estratificación socioeconómica. Un planteamiento general en este sentido se explorará en capítulos posteriores.

De los escenarios considerados surgen varios dilemas. Una primera opción que parte de una perspectiva nacional dentro del marco normativo actual resulta en cambios muy fuertes de asignación de estrato a los hogares bogotanos, lo que implicaría un largo período de transición para evitar fuertes incremento en la factura de SPD. Los otros dos modelos de estratificación basados en información catastral resultan de una clasificación de las viviendas tomando a Bogotá de manera independiente del país. La primera opción en este grupo se enmarcada en la normatividad actual (6 estratos), resulta en cambios en la distribución menos drástica que el modelo nacional, los errores de inclusión mejoran con respecto a la estratificación vigente, pero la probabilidad de asignarle a las viviendas estratos 2 y 3 sigue siendo muy alta respecto a los demás estratos, es decir, los errores de inclusión siguen siendo evidentes y significativos. Con el modelo que permite definir el número de 
estratos endógenamente (en este caso resultaron 9), se mejora en parte los errores de inclusión frente a tener 6 estratos, pero requeriría un cambio de la normatividad que permita ir más allá del número de grupos que determina la ley y no termina de resolver los principales problemas de la metodología que se tiene hoy en día. 